\title{
Finite Element Error Estimation for Quasi-Newtonian Fluid-Structure Interaction Problems
}

\author{
Hyesuk Lee $^{*} \quad$ Shuhan $\mathrm{Xu}^{\dagger}$
}

\begin{abstract}
We consider a monolithic scheme for fluid-structure interaction problems involving an incompressible quasi-Newtonian fluid. The monolithic formulation is obtained using the Arbitrary Lagrangian Eulerian (ALE) method with matching conditions at the fluid-structure interface. The stability and error analysis are performed for the finite element approximation. Finally, some numerical experiments that confirm the theoretical analysis are presented.
\end{abstract}

Key words fluid-structure interaction, monolithic approach, finite element error estimate

\section{Introduction}

Fluid-structure interaction (FSI) problems are multi-physics problems where surrounding or internal fluid flows interact with moveable or deformable structures. Such problems have various applications in engineering and biology, including interactions of airflow around the wings of micro-air vehicles, exploding gases in pipelines, and blood flow through arteries. In these interaction problems, the fluid and structure mutually influence each other; the fluid follows the motion of the structure while it also exerts stress to deform the structure. Specifically, this mutual influence implies that structure displacement determines the fluid domain, and the fluid velocity matches the structure velocity at their interface. The study of FSI problems also requires numerical simulations because laboratory experiments are limited in scope and analytical solutions are not available due to the complexity of FSI equations.

Various procedures have been developed to solve FSI problems, and they can be classified broadly into two methods: the monolithic approach and the partitioned approach. Both have their own advantages and disadvantages with regard to efficiency and stability. As the name implies, the monolithic approach solves the entire problem with one complex system, where the fluid and structure dynamics are treated in the same framework, and the interface conditions are implicit. This approach is an active area of research due to

\footnotetext{
*Department of Mathematical Sciences, Clemson University, Clemson, SC 29634-0975 (shuhanx@clemson.edu), Partially supported by the NSF under grants DMS-1016182, DMS-1418960.

${ }^{\dagger}$ Department of Mathematical Sciences, Clemson University, Clemson, SC 29634-0975 (hklee@clemson.edu), Partially supported by the NSF under grants DMS-1016182, DMS-1418960.
} 
its potential stability and accuracy. However, the complexity of the equation makes both analysis and computation difficult.

The Arbitrary Lagrangian Eulerian (ALE) method was first proposed in the 1980's [6, 12] and has been widely used thereafter. Nobile employed the ALE method to simulate an FSI problem consisting of a Navier-Stokes fluid and an elastic structure [19]. Related works on various boundary conditions $[7,9,20]$ and stability investigation $[5,9]$ were published in following years. A similar technique was also used for Stokes fluid-structure by Martin et. al. [17]. The ALE method considered in this work concerns the position of the fluid-structure interface where a fixed reference domain is mapped to a current moving domain. With an invertible and sufficiently regular ALE mapping, we can easily obtain a conforming mesh at arbitrary time following the interface movement as the image of a fixed mesh in the reference domain. Thus, the time derivative can be rewritten in a constant ALE coordinates, while the spatial terms are left in Eulerian coordinates.

Reports of both numerical experiments and analysis for a non-Newtonian FSI are much less common than the Newtonian case. In simulations for blood flow through vessels, a Newtonian (Stokes or Navier-Stokes) fluid has been used for most cases [4, 8]. However, it is inaccurate to model blood flow as a Newtonian fluid due to biological complexity. Several investigations have shown the significance of the non-Newtonian characteristics of blood flow $[2,3,13,16,18]$, by which this work has been motivated in focusing on the quasi-Newtonian case and investigating both analysis and numerical experiments of that case.

In this work, we consider a finite element approximation of the system of a quasiNewtonian fluid and a linear elastic structure. Compared to the analysis performed by Grandmont [1], which is based on a decoupled finite element approximation and a semiimplicit time-stepping strategy, our analysis and corresponding numerical tests are based on a monolithic scheme. The fluid equation is given in an Eulerian framework; thus, the fluid domain is time-dependent due to the movement of the interface. The isotropic linear elastic structure, on the other hand, is described in a Lagrangian frame of reference, giving the structure a fixed domain. With interface conditions and appropriately chosen function spaces, we obtain the monolithic global formulation for the FSI problem that accounts for the fluid and the structure at the same time.

The paper is organized as follows. Section 2 introduces the coupled fluid-structure system with initial and boundary conditions. The matching conditions for the two dynamics on the interface are also provided in this section. In Section 3, we provide a monolithic weak formulation in ALE frame. Finite element approximation and corresponding analysis are presented in Section 4. Section 5 presents the numerical experiments that confirm theoretical results, and finally, our results are summarized in Section 6 .

\section{Model Description}

Let $\Omega_{t}^{f}$ be the moving fluid domain at time $t$ in $\mathbb{R}^{2}$ with the boundary $\Gamma_{t}^{f}:=\Gamma_{D}^{f} \cup \Gamma_{I_{t}}$, where $\Gamma_{I_{t}}$ is the moving interface boundary. Let $\Omega^{s}$ be a fixed domain for the structure, described in Lagrangian terms. The boundary of structure is denoted as $\Gamma_{t}^{s}:=\Gamma_{N}^{s} \cup \Gamma_{D}^{s} \cup \Gamma_{I_{t}}$. We consider the system of a quasi-Newtonian flow and an isotropic linear elastic structure. 


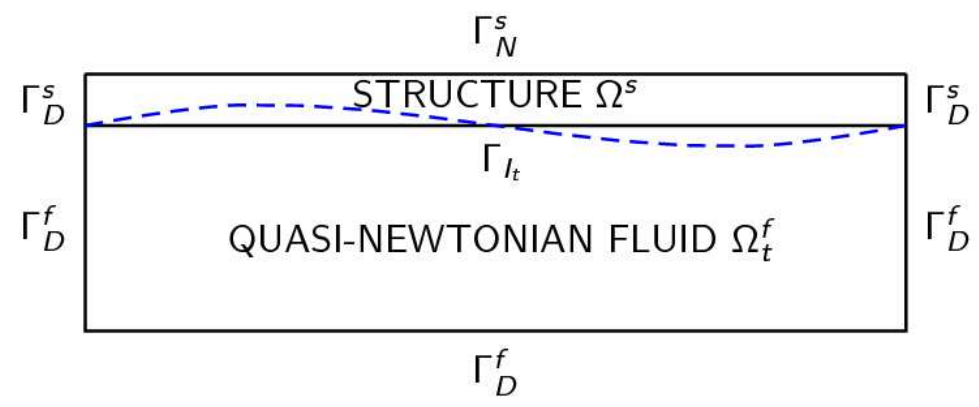

Figure 1: Fluid-Structure interaction domain

$$
\begin{aligned}
\rho_{f}\left(\frac{\partial \mathbf{u}}{\partial t}+\mathbf{u} \cdot \nabla \mathbf{u}\right)-\nabla \cdot \nu_{f}(|\mathbf{D}(\mathbf{u})|) \mathbf{D}(\mathbf{u})+\nabla p & =\mathbf{f}_{f} \quad \text { in } \Omega_{t}^{f}, \\
\nabla \cdot \mathbf{u} & =0 \quad \text { in } \Omega_{t}^{f}, \\
\rho_{s} \frac{\partial^{2} \boldsymbol{\eta}}{\partial t^{2}}-2 \nu_{s} \nabla \cdot \mathbf{D}(\boldsymbol{\eta})-\lambda \nabla(\nabla \cdot \boldsymbol{\eta}) & =\mathbf{f}_{s} \quad \text { in } \Omega^{s},
\end{aligned}
$$

where $\mathbf{u}$ denotes the velocity vector, $p$ the pressure of fluid, $\eta$ the displacement of structure, $\rho_{f}$ and $\rho_{s}$ the densities of the fluid and structure, respectively. In (2.1) and $(2.3), \mathbf{D}(\mathbf{u}):=$ $\left(\nabla \mathbf{u}+\nabla \mathbf{u}^{T}\right) / 2$ is the rate of the strain tensor, and $\mathbf{f}_{f}$ and $\mathbf{f}_{s}$ are the body forces. $\nu_{s}$ and $\lambda$ are the Lamé parameters defined as

$$
\nu_{s}=\frac{E}{2\left(1+\nu_{0}\right)}, \quad \lambda=\frac{\nu_{0} E}{\left(1-2 \nu_{0}\right)\left(1+\nu_{0}\right)},
$$

where $E$ is the Young's Modulus of the structure and $\nu_{0}$ is its Poisson ratio.

Initial and boundary conditions for $\mathbf{u}$ and $\boldsymbol{\eta}$ are given as follows

$$
\begin{aligned}
\mathbf{u}(\mathbf{x}, 0) & =\mathbf{u}_{0} \quad \text { in } \Omega_{0}^{f}, \\
\boldsymbol{\eta}(\mathbf{x}, 0) & =\boldsymbol{\eta}_{0}, \quad \boldsymbol{\eta}_{t}(\mathbf{x}, 0)=\dot{\boldsymbol{\eta}}_{0} \quad \text { in } \Omega^{s}, \\
\mathbf{u} & =\mathbf{0} \quad \text { on } \Gamma_{D}^{f}, \\
2 \nu_{s} \mathbf{D}(\boldsymbol{\eta}) \mathbf{n}_{s}+\lambda(\nabla \cdot \boldsymbol{\eta}) \mathbf{n}_{s} & =\mathbf{0} \quad \text { on } \Gamma_{N}^{s}, \\
\boldsymbol{\eta} & =\mathbf{0} \quad \text { on } \Gamma_{D}^{s},
\end{aligned}
$$

where $\mathbf{n}_{f}$ and $\mathbf{n}_{s}$ are the outward unit normal vectors to $\Omega_{t}^{f}$ and $\Omega^{s}$, respectively. The moving boundary $\Gamma_{I_{t}}$ is determined by the displacement $\boldsymbol{\eta}$ at time $t$ (Figure 1).

Based on the continuity of the velocity and the stress force, the matching conditions for the interface between the fluid and structure domains are

$$
\begin{aligned}
\frac{\partial \boldsymbol{\eta}}{\partial t} & =\mathbf{u} \text { on } \Gamma_{I_{t}}, \\
\left(\nu_{f}(|\mathbf{D}(\mathbf{u})|) \mathbf{D}(\mathbf{u})-p\right) \mathbf{n}_{f} & =-\left(2 \nu_{s} \mathbf{D}(\boldsymbol{\eta})+\lambda(\nabla \cdot \boldsymbol{\eta})\right) \mathbf{n}_{s} \text { on } \Gamma_{I_{t}} .
\end{aligned}
$$


The quasi-Newtonian fluid model (2.1) has a non-constant viscosity, which is a function of the magnitude of the deformation tensor. $\nu(|\mathbf{D}(\mathbf{u})|$ is a general viscosity function satisfying particular continuity and monotonicity properties. Typical models for such viscosity functions include the following:

Carreau model

$$
\nu_{f}(|\mathbf{D}(\mathbf{u})|)=\nu_{0}+\nu_{1}\left(1+|\mathbf{D}(\mathbf{u})|^{2}\right)^{\frac{r-2}{2}}, \text { where } \quad r>1, \nu_{0}, \nu_{1}>0,
$$

Ladyzhenskaya model

$$
\nu_{f}(|\mathbf{D}(\mathbf{u})|)=\nu_{0}+\nu_{1}|\mathbf{D}(\mathbf{u})|^{r-2}, \text { where } \quad r>1, \nu_{0}, \nu_{1}>0
$$

For the nonlinear function $\nu(|\mathbf{D}(\mathbf{u})|) \mathbf{D}(\mathbf{u})$, we make the following assumptions:

$$
\begin{aligned}
(\nu(|\boldsymbol{\sigma}|) \boldsymbol{\sigma}-\nu(|\boldsymbol{\tau}|) \boldsymbol{\tau}):(\boldsymbol{\sigma}-\boldsymbol{\tau}) \geq K_{1}|\boldsymbol{\sigma}-\boldsymbol{\tau}|^{2}, & \forall \boldsymbol{\sigma}, \boldsymbol{\tau} \in \mathbb{R}^{2 \times 2} \\
|(\nu(|\boldsymbol{\sigma}|) \boldsymbol{\sigma}-\nu(|\boldsymbol{\tau}|) \boldsymbol{\tau})| \leq K_{2}(|\boldsymbol{\sigma}|+|\boldsymbol{\tau}|)^{r-2}|\boldsymbol{\sigma}-\boldsymbol{\tau}|, & \forall \boldsymbol{\sigma}, \boldsymbol{\tau} \in \mathbb{R}^{2 \times 2}
\end{aligned}
$$

These properties imply that $\nu(|\cdot|)$ is strongly monotone and Lipschitz continuous for the bounded arguments. In addition, the models also satisfy

$$
\|(\nu(|\boldsymbol{\sigma}|) \boldsymbol{\sigma}-\nu(|\boldsymbol{\tau}|) \boldsymbol{\tau})\|_{0, \Omega_{f}^{f}} \leq K_{3}\|\boldsymbol{\sigma}-\boldsymbol{\tau}\|_{0, \Omega_{t}^{f}}, \quad \forall \boldsymbol{\sigma}, \boldsymbol{\tau} \in \mathbf{L}^{r}\left(\Omega_{t}^{f}\right),
$$

where $1<r \leq 2$. We consider the shear-thining case $(1<r<2)$ for which the velocity is assumed to be an $\mathbf{H}^{1}$ function.

\section{The ALE Formulation}

In most fluid-structure interaction problems, fluid and structure equations are posed from different perspectives in continuum mechanics: the Eulerian frame of reference for the fluid equations, and the Lagrangian frame of reference for the structure equations. The ALE [6] method allows the coupled problem to be posed in one framework, and therefore is widely used in simulating fluid flows in a moving domain.

With introduction of a family of time-dependent mappings from a fixed reference domain to the physical moving domain, the fluid equations can be rewritten in ALE formulation with respect to the reference domain. In this work, let the initial domain configuration $\Omega_{0}$ be the reference domain. Then for any time $t \in(0, T]$, we define a bijective mapping $\Psi_{t}$, which maps the reference domain $\Omega_{0}^{f}$ to the physical domain $\Omega_{t}^{f}$,

$$
\Psi_{t}: \Omega_{0}^{f} \rightarrow \Omega_{t}^{f}, \quad \Psi_{t}(\mathbf{y})=\mathbf{x}(\mathbf{y}, t)
$$

where $\mathbf{x}$ and $\mathbf{y}$ are the spatial coordinates in $\Omega_{t}^{f}$ and $\Omega_{0}^{f}$, respectively. We refer to $\mathbf{x}$ as the Eulerian coordinate and to $\mathbf{y}$ as the ALE coordinate. Assuming that $\Psi_{t}$ is invertible and $\Psi_{t}^{-1}$ is continuous, the ALE mapping introduces one-to-one coordinate transformations for the domains. For each time step, after determining the transformation function $\Psi_{t}$, the problem turns into a numerical simulation for a fluid defined on a fixed domain, which we are familiar with. 
For any function $\phi: \Omega_{t}^{f} \times[0, T] \rightarrow \mathbb{R}$ posed on the Eulerian frame, we may define the corresponding function $\bar{\phi}=\phi \circ \Psi_{t}$ on the ALE frame as

$$
\bar{\phi}: \Omega_{0} \rightarrow \mathbb{R}, \quad \bar{\phi}(\mathbf{y}, t)=\phi\left(\Psi_{t}(\mathbf{y}), t\right) .
$$

In the meantime, the corresponding time derivative on the ALE frame is defined as

$$
\left.\frac{\partial \phi}{\partial t}\right|_{\mathbf{y}}: \Omega_{t} \times[0, T] \rightarrow \mathbb{R},\left.\quad \frac{\partial \phi}{\partial t}\right|_{\mathbf{y}}(\mathbf{x}, t)=\frac{\partial \bar{\phi}}{\partial t}(\mathbf{y}, t)
$$

Using the above notation, the domain velocity can then be defined as $\mathbf{z}:=\left.\frac{\partial \mathbf{x}}{\partial t}\right|_{\mathbf{y}}$, which is actually the time derivative of Eulerian coordinate. Notice that $\mathbf{z}$ gives the velocity of each mesh node when discretized, so it is also called the mesh velocity.

Applying the chain rule, the ALE derivative of $\phi$ can be computed as

$$
\left.\frac{\partial \phi}{\partial t}\right|_{\mathbf{y}}=\left.\frac{\partial \phi}{\partial t}\right|_{\mathbf{x}}+\mathbf{z} \cdot \nabla_{\mathbf{x}} \phi
$$

Hence, the time derivative term on the Eulerian frame can be replaced by the ALE derivative

$$
\left.\frac{\partial \phi}{\partial t}\right|_{\mathbf{x}}=\left.\frac{\partial \phi}{\partial t}\right|_{\mathbf{y}}-\mathbf{z} \cdot \nabla_{\mathbf{x}} \phi
$$

We obtain the ALE formulation for the quasi-Newtonian flow equations (2.1)-(2.2) as

$$
\begin{aligned}
\rho_{f}\left(\left.\frac{\partial \mathbf{u}}{\partial t}\right|_{\mathbf{y}}+(\mathbf{u}-\mathbf{z}) \cdot \nabla_{\mathbf{x}} \mathbf{u}\right)-\nabla_{\mathbf{x}} \cdot \nu_{f}\left(\left|\mathbf{D}_{\mathbf{x}}(\mathbf{u})\right|\right) \mathbf{D}_{\mathbf{x}}(\mathbf{u})+\nabla_{\mathbf{x}} p & =\mathbf{f}_{f} \quad \text { in } \Omega_{t}^{f} \\
\nabla_{\mathbf{x}} \cdot \mathbf{u} & =0 \quad \text { in } \Omega_{t}^{f} .
\end{aligned}
$$

The time derivative term in (3.6) is now represented on the ALE frame, which can be computed in the fixed reference domain. However, all space derivative terms, including the divergence operator, remain in respect to the Eulerian coordinate $\mathbf{x}$, which has a simpler expression. To simplify notation, if not specified, $\mathbf{D}(\cdot)$ and $\nabla$ refer to $\mathbf{D}_{\mathbf{x}}(\cdot)$ and $\nabla_{\mathbf{x}}$, respectively, throughout this thesis.

One way to define the ALE mapping $\Psi_{t}$ is the harmonic extension technique [8]. This name comes from the fact that we extend the boundary position function $\mathbf{h}_{t}: \Gamma_{I_{0}} \times[0, T] \rightarrow$ $\Gamma_{I_{t}}$ into the whole domain. To compute the ALE mapping for our problem, we solve the Laplace equation

$$
\begin{cases}\Delta_{\mathbf{y}} \Psi_{t}(\mathbf{y})=0 & \text { in } \Omega_{0}^{f} \\ \Psi_{t}(\mathbf{y})=\mathbf{h}_{t}(\mathbf{y}) & \text { on } \Gamma_{I_{0}}\end{cases}
$$

with the boundary position function $\mathbf{h}_{t}: \partial \Omega_{0}^{f} \rightarrow \partial \Omega_{t}^{f}$ defined by

$$
\mathbf{h}_{t}(\mathbf{y})= \begin{cases}\mathbf{y}+\boldsymbol{\eta} & \text { on } \Gamma_{I_{t}} \\ \mathbf{y} & \text { on } \Gamma_{D}^{f}\end{cases}
$$

where $\boldsymbol{\eta}$ is the displacement of the moving interface. 
For the variational formulation of the flow equations (2.1)-(2.2) in the ALE framework, define function spaces for the reference domain as

$$
\begin{aligned}
& \mathbf{U}_{0}:=\left\{\overline{\mathbf{v}} \in \mathbf{H}^{1}\left(\Omega_{0}^{f}\right): \overline{\mathbf{v}}=\mathbf{0} \text { on } \Gamma_{D}^{f}\right\}, \\
& Q_{0}:=L^{2}\left(\Omega_{0}^{f}\right) .
\end{aligned}
$$

The function spaces for physical domain $\Omega_{t}^{f}$ are then defined as

$$
\begin{aligned}
& \mathbf{U}_{t}:=\left\{\mathbf{v}: \Omega_{t}^{f} \times[0, T] \rightarrow \mathbb{R}^{2}, \mathbf{v}=\overline{\mathbf{v}} \circ \Psi_{t}^{-1} \text { for } \overline{\mathbf{v}} \in \mathbf{U}_{0}\right\}, \\
& Q_{t}:=\left\{q: \Omega_{t}^{f} \times[0, T] \rightarrow \mathbb{R}, q=\bar{q} \circ \Psi_{t}^{-1} \text { for } \bar{p} \in Q_{0}\right\} .
\end{aligned}
$$

For the structure equation, the function space is defined as

$$
\mathbf{S}:=\left\{\boldsymbol{\xi} \in \mathbf{H}^{1}\left(\Omega^{s}\right): \boldsymbol{\xi}=\mathbf{0} \text { on } \Gamma_{D}^{s}\right\} .
$$

The variational formulation of (3.6)-(3.7) and (2.3) in the ALE framework can then be written as

$$
\begin{aligned}
& \rho_{f}\left(\left.\frac{\partial \mathbf{u}}{\partial t}\right|_{\mathbf{y}}+(\mathbf{u}-\mathbf{z}) \cdot \nabla \mathbf{u}, \mathbf{v}\right)_{\Omega_{t}^{f}}+\left(\nu_{f}(|\mathbf{D}(\mathbf{u})|) \mathbf{D}(\mathbf{u}), \mathbf{D}(\mathbf{v})\right)_{\Omega_{t}^{f}}-(p, \nabla \cdot \mathbf{v})_{\Omega_{t}^{f}} \\
& =\left(\mathbf{f}_{f}, \mathbf{v}\right)_{\Omega_{t}^{f}}+\left(\left(\nu_{f}(|\mathbf{D}(\mathbf{u})|) \mathbf{D}(\mathbf{u})-p\right) \cdot \mathbf{n}_{f}, \mathbf{v}\right)_{\Gamma_{I_{t}}} \quad \forall \mathbf{v} \in \mathbf{U}_{t} \\
& (q, \nabla \cdot \mathbf{u})_{\Omega_{t}^{f}}=0 \quad \forall q \in Q_{t}, \\
& \rho_{s}\left(\frac{\partial^{2} \boldsymbol{\eta}}{\partial t^{2}}, \boldsymbol{\xi}\right)_{\Omega^{s}}+2 \nu_{s}(\mathbf{D}(\boldsymbol{\eta}), \mathbf{D}(\boldsymbol{\xi}))_{\Omega^{s}}+\lambda(\nabla \cdot \boldsymbol{\eta}, \nabla \cdot \boldsymbol{\xi})_{\Omega^{s}} \\
& =\left(\mathbf{f}_{s}, \boldsymbol{\xi}\right)_{\Omega^{s}}+\left(\left(2 \nu_{s} D(\boldsymbol{\eta})+\lambda(\nabla \cdot \boldsymbol{\eta})\right) \mathbf{n}_{s}, \boldsymbol{\xi}\right)_{\Gamma_{I_{0}}} \quad \forall \boldsymbol{\xi} \in \mathbf{S}
\end{aligned}
$$

Define the function space for the coupled problem as

$$
\tilde{\mathbf{U}}_{t} \times \tilde{\mathbf{S}}:=\left\{(\mathbf{v}, \boldsymbol{\xi}) \in \mathbf{U}_{t} \times \mathbf{S}:\left.\mathbf{v}\right|_{\Gamma_{I_{t}}}=\left.\left(\frac{\partial \boldsymbol{\xi}}{\partial t} \circ \Psi_{t}^{-1}\right)\right|_{\Gamma_{I_{t}}}\right\},
$$

where the interface condition (2.9) is satisfied. Then, using (2.10), the boundary integral term in the right side of (3.11) can be substituted with $-\left(\left(\nu_{f}(|D(\mathbf{u})|) D(\mathbf{u})-p\right) \cdot \mathbf{n}_{f}, \boldsymbol{\xi} \circ \Psi_{t}^{-1}\right)_{\Gamma_{I_{t}}}$. Hence, combining (3.9)-(3.10) with ((3.11), we obtain a monolithic formulation in the ALE frame for the FSI system: find $(\mathbf{u}, p, \boldsymbol{\eta}) \in \tilde{\mathbf{U}}_{t} \times Q_{t} \times \tilde{\mathbf{S}}$ such that

$$
\begin{aligned}
& \rho_{s}\left(\frac{\partial^{2} \boldsymbol{\eta}}{\partial t^{2}}, \boldsymbol{\xi}\right)_{\Omega^{s}}+2 \nu_{s}(\mathbf{D}(\boldsymbol{\eta}), \mathbf{D}(\boldsymbol{\xi}))_{\Omega^{s}}+\lambda(\nabla \cdot \boldsymbol{\eta}, \nabla \cdot \boldsymbol{\xi})_{\Omega^{s}} \\
& +\rho_{f}\left[\left(\left.\frac{\partial \mathbf{u}}{\partial t}\right|_{\mathbf{y}}, \mathbf{v}\right)_{\Omega_{t}^{f}}+((\mathbf{u}-\mathbf{z}) \cdot \nabla \mathbf{u}, \mathbf{v})_{\Omega_{t}^{f}}\right] \\
& +\left(\nu_{f}(|\mathbf{D}(\mathbf{u})|) \mathbf{D}(\mathbf{u}), \mathbf{D}(\mathbf{v})\right)_{\Omega_{t}^{f}}-(p, \nabla \cdot \mathbf{v})_{\Omega_{t}^{f}}+(q, \nabla \cdot \mathbf{u})_{\Omega_{t}^{f}} \\
= & \left(\mathbf{f}_{f}, \mathbf{v}\right)_{\Omega_{t}^{f}}+\left(\mathbf{f}_{s}, \xi\right)_{\Omega^{s}} \quad \forall(\mathbf{v}, q, \boldsymbol{\xi}) \in \tilde{\mathbf{U}}_{t} \times Q_{t} \times \tilde{\mathbf{S}} .
\end{aligned}
$$

For purpose of analysis, we introduce a trilinear operator $\theta(\cdot, \cdot, \cdot)$

$$
\theta(\mathbf{u}, \mathbf{v}, \mathbf{w})_{\Omega_{t}^{f}}:=\frac{1}{2}\left[(\mathbf{u} \cdot \nabla \mathbf{v}, \mathbf{w})_{\Omega_{t}^{f}}-(\mathbf{u} \cdot \nabla \mathbf{w}, \mathbf{v})_{\Omega_{t}^{f}}\right] .
$$


Lemma 3.1 The trilinear operator $\theta(\cdot, \cdot, \cdot)$ has the following properties [11]:

$$
\begin{array}{ll}
\text { (i) } & \theta(\mathbf{u}, \mathbf{v}, \mathbf{v})_{\Omega_{t}^{f}}=0 \\
\text { (ii) } & \theta(\mathbf{u}, \mathbf{v}, \mathbf{w})_{\Omega_{t}^{f}} \leq C\|\mathbf{D}(\mathbf{u})\|_{0, \Omega_{t}^{f}}\|\mathbf{D}(\mathbf{v})\|_{0, \Omega_{t}^{f}}\|\mathbf{D}(\mathbf{w})\|_{0, \Omega_{t}^{f}}, \\
\text { (iii) } & \theta(\mathbf{u}, \mathbf{v}, \mathbf{w})_{\Omega_{t}^{f}} \leq C\|\mathbf{u}\|_{0, \Omega_{t}^{f}}^{\frac{1}{2}}\|\mathbf{D}(\mathbf{u})\|_{0, \Omega_{t}^{f}}^{\frac{1}{2}}\|\mathbf{D}(\mathbf{v})\|_{0, \Omega_{t}^{f}}\|\mathbf{D}(\mathbf{w})\|_{0, \Omega_{t}^{f}} .
\end{array}
$$

By Green's theorem we have

$$
\begin{aligned}
(\mathbf{u} \cdot \nabla \mathbf{u}, \mathbf{v})_{\Omega_{t}^{f}} & =-(\mathbf{u} \cdot \nabla \mathbf{v}, \mathbf{u})_{\Omega_{t}^{f}}-((\nabla \cdot \mathbf{u}) \mathbf{v}, \mathbf{u})_{\Omega_{t}^{f}}+\left(\left(\mathbf{u} \cdot \mathbf{n}_{f}\right) \mathbf{v}, \mathbf{u}\right)_{\Gamma_{I_{t}}}, \\
(\mathbf{z} \cdot \nabla \mathbf{u}, \mathbf{v})_{\Omega_{t}^{f}} & =-(\mathbf{z} \cdot \nabla \mathbf{v}, \mathbf{u})_{\Omega_{t}^{f}}-((\nabla \cdot \mathbf{z}) \mathbf{v}, \mathbf{u})_{\Omega_{t}^{f}}+\left(\left(\mathbf{z} \cdot \mathbf{n}_{f}\right) \mathbf{v}, \mathbf{u}\right)_{\Gamma_{I_{t}}}
\end{aligned}
$$

using $\nabla \cdot \mathbf{u}=0$ and (3.18),

$$
\begin{aligned}
(\mathbf{u} \cdot \nabla \mathbf{u}, \mathbf{v})_{\Omega_{t}^{f}} & =\frac{1}{2}(\mathbf{u} \cdot \nabla \mathbf{u}, \mathbf{v})_{\Omega_{t}^{f}}+\frac{1}{2}(\mathbf{u} \cdot \nabla \mathbf{u}, \mathbf{v})_{\Omega_{t}^{f}} \\
& =\frac{1}{2}\left[(\mathbf{u} \cdot \nabla \mathbf{u}, \mathbf{v})_{\Omega_{t}^{f}}-(\mathbf{u} \cdot \nabla \mathbf{v}, \mathbf{u})_{\Omega_{t}^{f}}+\left(\left(\mathbf{u} \cdot \mathbf{n}_{f}\right) \mathbf{v}, \mathbf{u}\right)_{\Gamma_{I_{t}}}\right] \\
& =\theta(\mathbf{u}, \mathbf{u}, \mathbf{v})_{\Omega_{t}^{f}}+\frac{1}{2}\left(\left(\mathbf{u} \cdot \mathbf{n}_{f}\right) \mathbf{v}, \mathbf{u}\right)_{\Gamma_{I_{t}}}
\end{aligned}
$$

Similarly,

$$
(\mathbf{z} \cdot \nabla \mathbf{u}, \mathbf{v})_{\Omega_{t}^{f}}=\theta(\mathbf{z}, \mathbf{u}, \mathbf{v})_{\Omega_{t}^{f}}-\frac{1}{2}((\nabla \cdot \mathbf{z}) \mathbf{v}, \mathbf{u})_{\Omega_{t}^{f}}+\frac{1}{2}\left(\left(\mathbf{z} \cdot \mathbf{n}_{f}\right) \mathbf{v}, \mathbf{u}\right)_{\Gamma_{I_{t}}}
$$

The interface condition (2.9) states that $\mathbf{u}=\frac{\partial \boldsymbol{\eta}}{\partial t}=\mathbf{z}$ on the interface, which implies

$$
\frac{1}{2}\left(\left(\mathbf{u} \cdot \mathbf{n}_{f}\right) \mathbf{v}, \mathbf{u}\right)_{\Gamma_{I_{t}}}=\frac{1}{2}\left(\left(\mathbf{z} \cdot \mathbf{n}_{f}\right) \mathbf{v}, \mathbf{u}\right)_{\Gamma_{I_{t}}}
$$

Using (3.20)-(3.22), (3.13) can be rewritten as

$$
\begin{aligned}
& \rho_{s}\left(\frac{\partial^{2} \boldsymbol{\eta}}{\partial t^{2}}, \boldsymbol{\xi}\right)_{\Omega^{s}}+2 \nu_{s}(\mathbf{D}(\boldsymbol{\eta}), \mathbf{D}(\boldsymbol{\xi}))_{\Omega^{s}}+\lambda(\nabla \cdot \boldsymbol{\eta}, \nabla \cdot \boldsymbol{\xi})_{\Omega^{s}} \\
& +\rho_{f}\left[\left(\left.\frac{\partial \mathbf{u}}{\partial t}\right|_{\mathbf{y}}, \mathbf{v}\right)_{\Omega_{t}^{f}}+\frac{1}{2}(\mathbf{u} \nabla \cdot \mathbf{z}, \mathbf{v})_{\Omega_{t}^{f}}+\theta(\mathbf{u}, \mathbf{u}, \mathbf{v})_{\Omega_{t}^{f}}-\theta(\mathbf{z}, \mathbf{u}, \mathbf{v})_{\Omega_{t}^{f}}\right] \\
& +\left(\nu_{f}(|\mathbf{D}(\mathbf{u})|) \mathbf{D}(\mathbf{u}), \mathbf{D}(\mathbf{v})\right)_{\Omega_{t}^{f}}-(p, \nabla \cdot \mathbf{v})_{\Omega_{t}^{f}}+(q, \nabla \cdot \mathbf{u})_{\Omega_{t}^{f}} \\
= & \left(\mathbf{f}_{f}, \mathbf{v}\right)_{\Omega_{t}^{f}}+\left(\mathbf{f}_{s}, \boldsymbol{\xi}\right)_{\Omega^{s}} \quad \forall(\mathbf{v}, q, \boldsymbol{\xi}) \in \tilde{\mathbf{U}}_{t} \times Q_{t} \times \tilde{\mathbf{S}} .
\end{aligned}
$$

\section{Finite Element Discretization}

Define finite element spaces for the approximation of $(\mathbf{u}, p)$ in $\Omega_{0}^{f}$ as

$$
\begin{aligned}
& \mathbf{U}_{h, 0}:=\left\{\mathbf{v} \in \mathbf{U}_{0} \cap\left(C^{0}\left(\overline{\Omega_{0}^{f}}\right)\right)^{2}:\left.\mathbf{v}\right|_{K} \in P_{2}(K)^{2}, \forall K \in T_{h, 0}\right\}, \\
& Q_{h, 0}:=\left\{q \in Q_{0} \cap C^{0}\left(\overline{\Omega_{0}^{f}}\right):\left.q\right|_{K} \in P_{1}(K), \forall K \in T_{h, 0}\right\},
\end{aligned}
$$


where $T_{h, 0}$ is a triangulation satisfying the quasi-uniform mesh condition. It is well known that the Taylor-Hood pair $\left(P_{2}, P_{1}\right)$ satisfies the $L B B$ condition

$$
\inf _{0 \neq q_{h} \in Q_{h, 0}} \sup _{0 \neq \mathbf{v}_{h} \in \mathbf{U}_{h, 0}} \frac{\left(q_{h}, \nabla \cdot \mathbf{v}_{h}\right)}{\left\|\mathbf{v}_{h}\right\|_{1}\left\|q_{h}\right\|_{0}} \geq C,
$$

where $C$ is a positive constant independent of $h$. The finite element spaces for $\left(\mathbf{u}_{h}, p_{h}\right)$ in $\Omega_{t}^{f}$ are then defined as

$$
\begin{aligned}
\mathbf{U}_{h, t} & :=\left\{\mathbf{v}_{h}: \Omega_{t}^{f} \times[0, T] \rightarrow \mathbb{R}^{2}, \mathbf{v}_{h}=\overline{\mathbf{v}}_{h} \circ \Psi_{h, t}^{-1} \text { for } \overline{\mathbf{v}}_{h} \in \mathbf{U}_{h, 0}\right\}, \\
Q_{h, t} & :=\left\{q_{h}: \Omega_{t}^{f} \times[0, T] \rightarrow \mathbb{R}, q_{h}=\bar{q}_{h} \circ \Psi_{h, t}^{-1} \text { for } \bar{q}_{h} \in Q_{h, 0}\right\},
\end{aligned}
$$

where $\Psi_{h, t}: \Omega_{0} \rightarrow \Omega_{t}$ is a discrete mapping approximated by $P_{1}$ Lagrangian finite elements such that $\Psi_{h, t}(\mathbf{y})=\mathbf{x}_{h}(\mathbf{y}, t)$. For the discrete ALE mapping, define the space

$$
\mathbf{X}_{h}:=\left\{\mathbf{x} \in \mathbf{H}^{1}\left(\Omega_{0}\right):\left.\mathbf{x}\right|_{K} \in P_{1}(K)^{2}, \forall K \in T_{h, 0}\right\}
$$

The corresponding discrete domain velocity is then defined as $\mathbf{z}_{h}=\left.\frac{\partial \mathbf{x}_{h}}{\partial t}\right|_{\mathbf{y}}$ with the assumption that

$$
\max \left\{\left\|\mathbf{z}_{h}\right\|_{1, \infty, \Omega_{t}^{f}},\left\|\frac{\partial}{\partial t} \mathbf{z}_{h}\right\|_{1, \infty, \Omega_{t}}\right\} \leq M
$$

based on the regularity of ALE mapping [10, 17].

The finite element space for $\boldsymbol{\eta}_{h}$ is defined as

$$
\mathbf{S}_{h}:=\left\{\boldsymbol{\xi}_{h} \in \mathbf{S} \cap\left(C^{0}\left(\overline{\Omega^{s}}\right)\right)^{2}:\left.\boldsymbol{\xi}_{h}\right|_{K} \in P_{2}(K)^{2}, \forall K \in \bar{T}_{h}\right\}
$$

where $\bar{T}_{h}$ is a triangulation in the structure domain. Then with the discrete coupled function spaces

$$
\tilde{\mathbf{U}}_{h, t} \times \tilde{\mathbf{S}}_{h}:=\left\{\left(\mathbf{v}_{h}, \boldsymbol{\xi}_{h}\right) \in \mathbf{U}_{h, t} \times \mathbf{S}_{h}:\left.\mathbf{v}_{h}\right|_{\Gamma_{I_{t}}}=\left.\left(\frac{\partial \boldsymbol{\xi}_{h}}{\partial t} \circ \Psi_{h, t}^{-1}\right)\right|_{\Gamma_{I_{t}}}\right\}
$$

the semi-discrete variational formulation of (3.23) is written as

$$
\begin{aligned}
& \rho_{s}\left(\frac{\partial^{2} \boldsymbol{\eta}_{h}}{\partial t^{2}}, \boldsymbol{\xi}_{h}\right)_{\Omega^{s}}+2 \nu_{s}\left(\mathbf{D}\left(\boldsymbol{\eta}_{h}\right), \mathbf{D}\left(\boldsymbol{\xi}_{h}\right)\right)_{\Omega^{s}}+\lambda\left(\nabla \cdot \boldsymbol{\eta}_{h}, \nabla \cdot \boldsymbol{\xi}_{h}\right)_{\Omega^{s}} \\
& +\rho_{f}\left[\left(\left.\frac{\partial \mathbf{u}_{h}}{\partial t}\right|_{\mathbf{y}}, \mathbf{v}_{h}\right)_{\Omega_{t}^{f}}+\frac{1}{2}\left(\mathbf{u}_{h} \nabla \cdot \mathbf{z}_{h}, \mathbf{v}_{h}\right)_{\Omega_{t}^{f}}+\theta\left(\mathbf{u}_{h}, \mathbf{u}_{h}, \mathbf{v}_{h}\right)_{\Omega_{t}^{f}}-\theta\left(\mathbf{z}_{h}, \mathbf{u}_{h}, \mathbf{v}_{h}\right)_{\Omega_{t}^{f}}\right] \\
& +\left(\nu_{f}\left(\left|\mathbf{D}\left(\mathbf{u}_{h}\right)\right|\right) \mathbf{D}\left(\mathbf{u}_{h}\right), \mathbf{D}\left(\mathbf{v}_{h}\right)\right)_{\Omega_{t}^{f}}-\left(p_{h}, \nabla \cdot \mathbf{v}_{h}\right)_{\Omega_{t}^{f}}+\left(q_{h}, \nabla \cdot \mathbf{u}_{h}\right)_{\Omega_{t}^{f}} \\
= & \left(\mathbf{f}_{f}, \mathbf{v}_{h}\right)_{\Omega_{t}^{f}}+\left(\mathbf{f}_{s}, \boldsymbol{\xi}_{h}\right)_{\Omega^{s}} \quad \forall\left(\mathbf{v}_{h}, q_{h}, \boldsymbol{\xi}_{h}\right) \in \tilde{\mathbf{U}}_{h, t} \times Q_{h, t} \times \tilde{\mathbf{S}}_{h} .
\end{aligned}
$$

We define a discrete divergence free space as

$$
\tilde{\mathbf{V}}_{h, t}^{f}:=\left\{\mathbf{v}_{h} \in \tilde{\mathbf{U}}_{h, t}^{f}:\left(\nabla \cdot \mathbf{v}_{h}, q_{h}\right)_{\Omega_{t}^{f}}=0 \quad \forall q_{h} \in Q_{h, t}\right\},
$$

which will be used in the proof of Theorem 4.2. 
Theorem 4.1 A solution to the semi-discrete problem (4.5) satisfies the estimate

$$
\begin{aligned}
& \frac{\rho_{s}}{2}\left\|\frac{\partial \boldsymbol{\eta}_{h}}{\partial t}\right\|_{0, \Omega^{s}}^{2}+\nu_{s}\left\|D\left(\boldsymbol{\eta}_{h}\right)\right\|_{0, \Omega^{s}}^{2}+\frac{\lambda}{2}\left\|\nabla \cdot \boldsymbol{\eta}_{h}\right\|_{0, \Omega^{s}}^{2}+\frac{\rho_{f}}{2}\left\|\mathbf{u}_{h}\right\|_{0, \Omega_{t}^{f}}^{2}+\frac{K_{1}}{2} \int_{0}^{t}\left\|D\left(\mathbf{u}_{h}\right)\right\|_{0, \Omega_{\tilde{t}}^{f}}^{2} d \tilde{t} \\
\leq & \frac{\rho_{s}}{2}\left\|\dot{\boldsymbol{\eta}}_{0}\right\|_{0, \Omega^{s}}^{2}+\nu_{s}\left\|D\left(\boldsymbol{\eta}_{0}\right)\right\|_{0, \Omega^{s}}^{2}+\frac{\lambda}{2}\left\|\nabla \cdot \boldsymbol{\eta}_{0}\right\|_{0, \Omega^{s}}^{2}+\frac{\rho_{f}}{2}\left\|\mathbf{u}_{0}\right\|_{0, \Omega_{0}^{f}}^{2} \\
& +C \int_{0}^{t}\left\|\mathbf{f}_{f}\right\|_{0, \Omega_{\tilde{t}}^{f}}^{2}+\left\|\mathbf{f}_{s}\right\|_{0, \Omega^{s}}^{2} d \tilde{t} .
\end{aligned}
$$

Proof: Set $\mathbf{v}_{h}=\mathbf{u}_{h}, q_{h}=p_{h}, \boldsymbol{\xi}_{h}=\frac{\partial \boldsymbol{\eta}_{h}}{\partial t}$ in (4.5). Using the Reynolds' transportation formula $[15,19]$, we have

$$
\begin{aligned}
\left(\left.\frac{\partial \mathbf{u}_{h}}{\partial t}\right|_{\mathbf{y}}, \mathbf{u}_{h}\right)_{\Omega_{t}^{f}} & =\frac{1}{2}\left(\left.\frac{\partial \mathbf{u}_{h}^{2}}{\partial t}\right|_{\mathbf{y}}, 1\right)_{\Omega_{t}^{f}} \\
& =\frac{1}{2}\left[\frac{\partial}{\partial t}\left(\mathbf{u}_{h}^{2}, 1\right)_{\Omega_{t}^{f}}-\left(\mathbf{u}_{h}^{2} \nabla \cdot \mathbf{z}_{h}, 1\right)_{\Omega_{t}^{f}}\right] \\
& =\frac{1}{2} \frac{\partial}{\partial t}\left\|\mathbf{u}_{h}\right\|_{0, \Omega_{t}^{f}}^{2}-\frac{1}{2}\left(\mathbf{u}_{h} \nabla \cdot \mathbf{z}_{h}, \mathbf{u}_{h}\right)_{\Omega_{t}^{f}}
\end{aligned}
$$

In the meantime,

$$
\begin{aligned}
& \rho_{s}\left(\frac{\partial^{2} \boldsymbol{\eta}_{h}}{\partial t^{2}}, \frac{\partial \boldsymbol{\eta}_{h}}{\partial t}\right)_{\Omega^{s}}+2 \nu_{s}\left(\mathbf{D}\left(\boldsymbol{\eta}_{h}\right), \mathbf{D}\left(\frac{\partial \boldsymbol{\eta}_{h}}{\partial t}\right)\right)_{\Omega^{s}}+\lambda\left(\nabla \cdot \boldsymbol{\eta}_{h}, \nabla \cdot \frac{\partial \boldsymbol{\eta}_{h}}{\partial t}\right)_{\Omega^{s}} \\
= & \frac{\partial}{\partial t}\left(\frac{\rho_{s}}{2}\left\|\frac{\partial \boldsymbol{\eta}_{h}}{\partial t}\right\|_{0, \Omega^{s}}^{2}+\nu_{s}\left\|\mathbf{D}\left(\boldsymbol{\eta}_{h}\right)\right\|_{0, \Omega^{s}}^{2}+\frac{\lambda}{2}\left\|\nabla \cdot \boldsymbol{\eta}_{h}\right\|_{0, \Omega^{s}}^{2}\right),
\end{aligned}
$$

while (3.15) implies that

$$
\theta\left(\mathbf{u}_{h}, \mathbf{u}_{h}, \mathbf{u}_{h}\right)_{\Omega_{t}^{f}}-\theta\left(\mathbf{z}_{h}, \mathbf{u}_{h}, \mathbf{u}_{h}\right)_{\Omega_{t}^{f}}=0 .
$$

By Poincaré inequality, the right hand side of (4.5) can be bounded by

$$
\begin{aligned}
& \left(\mathbf{f}_{f}, \mathbf{u}_{h}\right)_{\Omega_{t}^{f}}+\left(\mathbf{f}_{s}, \frac{\partial \boldsymbol{\eta}_{h}}{\partial t}\right)_{\Omega^{s}} \\
\leq & C\left(\epsilon_{1}\right)\left\|\mathbf{f}_{f}\right\|_{0, \Omega_{t}^{f}}^{2}+\epsilon_{1}\left\|\mathbf{D}\left(\mathbf{u}_{h}\right)\right\|_{0, \Omega_{t}^{f}}^{2}+C\left(\epsilon_{2}\right)\left\|\mathbf{f}_{s}\right\|_{0, \Omega^{s}}^{2}+\epsilon_{2}\left\|\frac{\partial \boldsymbol{\eta}_{h}}{\partial t}\right\|_{0, \Omega^{s}}^{2} .
\end{aligned}
$$

Applying (2.11), (4.7) - (4.10) in (4.5),

$$
\begin{aligned}
& \frac{\partial}{\partial t}\left(\frac{\rho_{s}}{2}\left\|\frac{\partial \boldsymbol{\eta}_{h}}{\partial t}\right\|_{0, \Omega^{s}}^{2}+\nu_{s}\left\|\mathbf{D}\left(\boldsymbol{\eta}_{h}\right)\right\|_{0, \Omega^{s}}^{2}+\frac{\lambda}{2}\left\|\nabla \cdot \boldsymbol{\eta}_{h}\right\|_{0, \Omega^{s}}^{2}+\frac{\rho_{f}}{2}\left\|\mathbf{u}_{h}\right\|_{0, \Omega_{t}^{f}}^{2}\right) \\
& +K_{1}\left\|\mathbf{D}\left(\mathbf{u}_{h}\right)\right\|_{0, \Omega_{t}^{f}}^{2} \\
\leq & C\left(\epsilon_{1}\right)\left\|\mathbf{f}_{f}\right\|_{0, \Omega_{t}^{f}}^{2}+\epsilon_{1}\left\|\mathbf{D}\left(\mathbf{u}_{h}\right)\right\|_{0, \Omega_{t}^{f}}^{2}+C\left(\epsilon_{2}\right)\left\|\mathbf{f}_{s}\right\|_{0, \Omega^{s}}^{2}+\epsilon_{2}\left\|\frac{\partial \boldsymbol{\eta}_{h}}{\partial t}\right\|_{0, \Omega^{s}}^{2} .
\end{aligned}
$$


Setting $\epsilon_{1}=\frac{K_{1}}{2}, \epsilon_{2}=\frac{\rho_{s}}{2},(4.11)$ can be reduced to:

$$
\begin{aligned}
& \frac{\partial}{\partial t}\left(\frac{\rho_{s}}{2}\left\|\frac{\partial \boldsymbol{\eta}_{h}}{\partial t}\right\|_{0, \Omega^{s}}^{2}+\nu_{s}\left\|\mathbf{D}\left(\boldsymbol{\eta}_{h}\right)\right\|_{0, \Omega^{s}}^{2}+\frac{\lambda}{2}\left\|\nabla \cdot \boldsymbol{\eta}_{h}\right\|_{0, \Omega^{s}}^{2}+\frac{\rho_{f}}{2}\left\|\mathbf{u}_{h}\right\|_{0, \Omega_{t}^{f}}^{2}\right) \\
& +\frac{K_{1}}{2}\left\|\mathbf{D}\left(\mathbf{u}_{h}\right)\right\|_{0, \Omega_{t}^{f}}^{2} \\
\leq & \frac{\rho_{s}}{2}\left\|\frac{\partial \boldsymbol{\eta}_{h}}{\partial t}\right\|_{0, \Omega^{s}}^{2}+C\left(\epsilon_{1}\right)\left\|\mathbf{f}_{f}\right\|_{0, \Omega_{t}^{f}}^{2}+C\left(\epsilon_{2}\right)\left\|\mathbf{f}_{s}\right\|_{0, \Omega^{s}}^{2} .
\end{aligned}
$$

The estimate (4.6) can then be obtained by Gronwall's Lemma.

We will present an a priori error estimate of the finite element solution in the following theorem.

Theorem 4.2 Suppose $(\mathbf{u}, p, \boldsymbol{\eta})$ is a solution of (2.1)-(2.8) and

$$
\mathbf{u} \in \mathbf{L}^{4}\left(0, T ; \mathbf{H}^{1}\left(\Omega_{t}^{f}\right)\right) .
$$

A solution to the semi-discrete problem (4.5) satisfies the error estimate

$$
\begin{gathered}
\rho_{s}\left\|\frac{\partial}{\partial t}\left(\boldsymbol{\eta}-\boldsymbol{\eta}_{h}\right)\right\|_{0, \Omega^{s}}^{2}+\nu_{s}\left\|\mathbf{D}\left(\boldsymbol{\eta}-\boldsymbol{\eta}_{h}\right)\right\|_{0, \Omega^{s}}^{2}+\frac{\lambda}{2}\left\|\nabla \cdot\left(\boldsymbol{\eta}-\boldsymbol{\eta}_{h}\right)\right\|_{0, \Omega^{s}}^{2} \\
+\rho_{f}\left\|\mathbf{u}-\mathbf{u}_{h}\right\|_{0, \Omega_{t}^{f}}^{2}+K_{1} \int_{0}^{t}\left\|\mathbf{D}\left(\mathbf{u}-\mathbf{u}_{h}\right)\right\|_{0, \Omega_{\tilde{t}}^{f}}^{2} d \tilde{t} \\
\leq C\left\{\left\|\mathbf{u}_{0}-\mathbf{u}_{h}(0)\right\|_{0, \Omega_{0}^{f}}^{2}+\left\|\mathbf{D}\left(\boldsymbol{\eta}_{0}-\boldsymbol{\eta}_{h}(0)\right)\right\|_{0, \Omega^{s}}^{2}+\left\|\mathbf{D}\left(\dot{\boldsymbol{\eta}}_{0}-\dot{\boldsymbol{\eta}}_{h}(0)\right)\right\|_{0, \Omega^{s}}^{2}\right. \\
+\tilde{\mathbf{u}}_{h} \tilde{p}_{h}, \tilde{\boldsymbol{\eta}}_{h} \in \tilde{\mathbf{U}}_{h, t} \times Q_{h, t} \times \tilde{\mathbf{S}}_{h} \\
+\left\|\mathbf{D}\left(\boldsymbol{\eta}-\tilde{\boldsymbol{\eta}}_{h}\right)\right\|_{0, \Omega^{s}}^{2}+\int_{0}^{t}\left\|\frac{\partial^{2}}{\partial \tilde{t}^{2}}\left(\boldsymbol{\eta}-\tilde{\boldsymbol{\eta}}_{h}\right)\right\|_{L^{4}\left(0, T ; H^{1}\right)}^{4}+\max _{0 \leq t \leq T}\left\|\mathbf{D}\left(\mathbf{u}-\tilde{\mathbf{u}}_{h}\right)\right\|_{0, \Omega_{t}^{f}}^{2}+\left\|\frac{\partial}{\partial \tilde{t}} \mathbf{D}\left(\boldsymbol{\eta}-\tilde{\boldsymbol{\eta}}_{h}\right)\right\|_{0, \Omega^{s}}^{2} \\
\left.\left.+\left\|\left.\frac{\partial\left(\mathbf{u}-\tilde{\mathbf{u}}_{h}\right)}{\partial \tilde{t}}\right|_{\mathbf{y}}\right\|_{0, \Omega_{\tilde{t}}^{f}}^{2}+\left\|\tilde{p}_{h}-p\right\|_{0, \Omega_{\tilde{t}}^{f}}^{2} d \tilde{t}\right]\right\} .
\end{gathered}
$$

Proof: Let $\tilde{\mathbf{u}}_{h}, \tilde{p}_{h}, \tilde{\boldsymbol{\eta}}_{h}$ be arbitrary functions in $\tilde{\mathbf{V}}_{h, t}, Q_{h, t}, \tilde{\mathbf{S}}_{h}$, respectively. Then we have the relations:

$$
\begin{array}{ccc}
\boldsymbol{\eta}-\boldsymbol{\eta}_{h}=\boldsymbol{\phi}-\boldsymbol{\psi} & \text { where } & \boldsymbol{\phi}=\tilde{\boldsymbol{\eta}}_{h}-\boldsymbol{\eta}_{h}, \boldsymbol{\psi}=\tilde{\boldsymbol{\eta}}_{h}-\boldsymbol{\eta}, \\
\mathbf{u}-\mathbf{u}_{h}=\mathbf{I}-\mathbf{g} & \text { where } & \mathbf{I}=\tilde{\mathbf{u}}_{h}-\mathbf{u}_{h}, \mathbf{g}=\tilde{\mathbf{u}}_{h}-\mathbf{u} .
\end{array}
$$

To simplify the analysis, we slightly modify (3.23) by taking the discrete domain velocity (i.e., replacing $\mathbf{z}$ by $\mathbf{z}_{h}$ ). The error estimate for $\left\|\mathbf{z}-\mathbf{z}_{h}\right\|_{1, \Omega_{t}^{f}}$ is shown in [10]. Assuming $\mathbf{z}$ is uniformly bounded and using (4.3), it is easily seen that the same error estimate result 
will hold if (3.23) is used without the replacement. Subtracting the semi-discretized weak formulation (4.5) from the continuous weak formulation (3.23) gives

$$
\begin{aligned}
& \rho_{s}\left(\frac{\partial^{2} \boldsymbol{\phi}}{\partial t^{2}}-\frac{\partial^{2} \boldsymbol{\psi}}{\partial t^{2}}, \boldsymbol{\xi}_{h}\right)_{\Omega^{s}}+2 \nu_{s}\left(\mathbf{D}(\boldsymbol{\phi})-\mathbf{D}(\boldsymbol{\psi}), \mathbf{D}\left(\boldsymbol{\xi}_{h}\right)\right)_{\Omega^{s}}+\lambda\left(\nabla \cdot \boldsymbol{\phi}-\nabla \cdot \boldsymbol{\psi}, \nabla \cdot \boldsymbol{\xi}_{h}\right)_{\Omega^{s}} \\
& +\rho_{f}\left(\left.\frac{\partial(\mathbf{I}-\mathbf{g})}{\partial t}\right|_{\mathbf{y}}, \mathbf{v}_{h}\right)_{\Omega_{t}^{f}}+\rho_{f}\left(\theta\left(\mathbf{u}, \mathbf{u}, \mathbf{v}_{h}\right)_{\Omega_{t}^{f}}-\theta\left(\mathbf{u}_{h}, \mathbf{u}_{h}, \mathbf{v}_{h}\right)_{\Omega_{t}^{f}}\right) \\
& +\rho_{f}\left[\frac{1}{2}\left((\mathbf{I}-\mathbf{g}) \nabla \cdot \mathbf{z}_{h}, \mathbf{v}_{h}\right)_{\Omega_{t}^{f}}-\theta\left(\mathbf{z}_{h}, \mathbf{I}-\mathbf{g}, \mathbf{v}_{h}\right)_{\Omega_{t}^{f}}\right] \\
& +\left(\nu_{f}(|\mathbf{D}(\mathbf{u})|) \mathbf{D}(\mathbf{u}), \mathbf{D}\left(\mathbf{v}_{h}\right)\right)_{\Omega_{t}^{f}}-\left(\nu_{f}\left(\left|\mathbf{D}\left(\mathbf{u}_{h}\right)\right|\right) \mathbf{D}\left(\mathbf{u}_{h}\right), \mathbf{D}\left(\mathbf{v}_{h}\right)\right)_{\Omega_{t}^{f}} \\
& -\left(p, \nabla \cdot \mathbf{v}_{h}\right)_{\Omega_{t}^{f}}+\left(p_{h}, \nabla \cdot \mathbf{v}_{h}\right)_{\Omega_{t}^{f}}-\left(q_{h}, \nabla \cdot \mathbf{u}_{h}\right)_{\Omega_{t}^{f}}+\left(q_{h}, \nabla \cdot \mathbf{u}\right)_{\Omega_{t}^{f}}=\mathbf{0} .
\end{aligned}
$$

Note that

$$
\begin{aligned}
& \theta\left(\mathbf{u}, \mathbf{u}, \mathbf{v}_{h}\right)_{\Omega_{t}^{f}}-\theta\left(\mathbf{u}_{h}, \mathbf{u}_{h}, \mathbf{v}_{h}\right)_{\Omega_{t}^{f}} \\
= & \theta\left(\mathbf{u}, \mathbf{u}, \mathbf{v}_{h}\right)_{\Omega_{t}^{f}}-\theta\left(\mathbf{u}_{h}, \mathbf{u}, \mathbf{v}_{h}\right)_{\Omega_{t}^{f}}+\theta\left(\mathbf{u}_{h}, \mathbf{u}, \mathbf{v}_{h}\right)_{\Omega_{t}^{f}}-\theta\left(\mathbf{u}_{h}, \mathbf{u}_{h}, \mathbf{v}_{h}\right)_{\Omega_{t}^{f}} \\
= & \theta\left(\mathbf{I}-\mathbf{g}, \mathbf{u}, \mathbf{v}_{h}\right)_{\Omega_{t}^{f}}+\theta\left(\mathbf{u}_{h}, \mathbf{I}-\mathbf{g}, \mathbf{v}_{h}\right)_{\Omega_{t}^{f}} \\
= & \theta\left(\mathbf{I}, \mathbf{u}, \mathbf{v}_{h}\right)_{\Omega_{t}^{f}}-\theta\left(\mathbf{g}, \mathbf{u}, \mathbf{v}_{h}\right)_{\Omega_{t}^{f}}+\theta\left(\mathbf{u}_{h}, \mathbf{I}, \mathbf{v}_{h}\right)_{\Omega_{t}^{f}}-\theta\left(\mathbf{u}_{h}, \mathbf{g}, \mathbf{v}_{h}\right)_{\Omega_{t}^{f}} .
\end{aligned}
$$

Using (4.15) and the fact that $\nabla \cdot \mathbf{u}=0$ for the strong solution, moving some terms to the right hand side of the equation and adding $\left(\nu_{f}\left(\left|\mathbf{D}\left(\tilde{\mathbf{u}}_{h}\right)\right|\right) \mathbf{D}\left(\tilde{\mathbf{u}}_{h}\right), \mathbf{D}\left(\mathbf{v}_{h}\right)\right)_{\Omega_{t}^{f}}-\left(\tilde{p}_{h}, \nabla \cdot \mathbf{v}_{h}\right)_{\Omega_{t}^{f}}$ to both sides, we obtain

$$
\begin{aligned}
& \rho_{s}\left(\frac{\partial^{2} \boldsymbol{\phi}}{\partial t^{2}}, \boldsymbol{\xi}_{h}\right)_{\Omega^{s}}+2 \nu_{s}\left(\mathbf{D}(\boldsymbol{\phi}), \mathbf{D}\left(\boldsymbol{\xi}_{h}\right)\right)_{\Omega^{s}}+\lambda\left(\nabla \cdot \boldsymbol{\phi}, \nabla \cdot \boldsymbol{\xi}_{h}\right)_{\Omega^{s}} \\
& +\rho_{f}\left(\left.\frac{\partial \mathbf{I}}{\partial t}\right|_{\mathbf{y}}, \mathbf{v}_{h}\right)_{\Omega_{t}^{f}}+\rho_{f} \theta\left(\mathbf{u}_{h}, \mathbf{I}, \mathbf{v}_{h}\right)_{\Omega_{t}^{f}}+\rho_{f}\left[\frac{1}{2}\left(\mathbf{I} \nabla \cdot \mathbf{z}_{h}, \mathbf{v}_{h}\right)_{\Omega_{t}^{f}}-\theta\left(\mathbf{z}_{h}, \mathbf{I}, \mathbf{v}_{h}\right)_{\Omega_{t}^{f}}\right] \\
& +\left(\nu_{f}\left(\left|\mathbf{D}\left(\tilde{\mathbf{u}}_{h}\right)\right|\right) \mathbf{D}\left(\tilde{\mathbf{u}}_{h}\right), \mathbf{D}\left(\mathbf{v}_{h}\right)\right)_{\Omega_{t}^{f}}-\left(\nu_{f}\left(\left|\mathbf{D}\left(\mathbf{u}_{h}\right)\right|\right) \mathbf{D}\left(\mathbf{u}_{h}\right), \mathbf{D}\left(\mathbf{v}_{h}\right)\right)_{\Omega_{t}^{f}} \\
& -\left(\tilde{p}_{h}-p_{h}, \nabla \cdot \mathbf{v}_{h}\right)_{\Omega_{t}^{f}} \\
= & \rho_{s}\left(\frac{\partial^{2} \boldsymbol{\psi}}{\partial t^{2}}, \boldsymbol{\xi}_{h}\right)_{\Omega^{s}}+2 \nu_{s}\left(\mathbf{D}(\boldsymbol{\psi}), \mathbf{D}\left(\boldsymbol{\xi}_{h}\right)\right)_{\Omega^{s}}+\lambda\left(\nabla \cdot \boldsymbol{\psi}, \nabla \cdot \boldsymbol{\xi}_{h}\right)_{\Omega^{s}} \\
& +\rho_{f}\left(\left.\frac{\partial \mathbf{g}}{\partial t}\right|_{\mathbf{y}}, \mathbf{v}_{h}\right)_{\Omega_{t}^{f}}+\rho_{f}\left[\theta\left(\mathbf{u}_{h}, \mathbf{g}, \mathbf{v}_{h}\right)_{\Omega_{t}^{f}}+\theta\left(\mathbf{g}, \mathbf{u}, \mathbf{v}_{h}\right)_{\Omega_{t}^{f}}-\theta\left(\mathbf{I}, \mathbf{u}, \mathbf{v}_{h}\right)_{\Omega_{t}^{f}}\right] \\
& +\rho_{f}\left[\frac{1}{2}\left(\mathbf{g} \nabla \cdot \mathbf{z}_{h}, \mathbf{v}_{h}\right)_{\Omega_{t}^{f}}-\theta\left(\mathbf{z}_{h}, \mathbf{g}, \mathbf{v}_{h}\right)_{\Omega_{t}^{f}}\right] \\
& +\left(\nu_{f}\left(\left|\mathbf{D}\left(\tilde{\mathbf{u}}_{h}\right)\right|\right) \mathbf{D}\left(\tilde{\mathbf{u}}_{h}\right), \mathbf{D}\left(\mathbf{v}_{h}\right)\right)_{\Omega_{t}^{f}}-\left(\nu_{f}(|\mathbf{D}(\mathbf{u})|) \mathbf{D}(\mathbf{u}), \mathbf{D}\left(\mathbf{v}_{h}\right)\right)_{\Omega_{t}^{f}} \\
& -\left(\tilde{p}_{h}-p, \nabla \cdot \mathbf{v}_{h}\right)_{\Omega_{t}^{f}}
\end{aligned}
$$


In (4.16) set $\boldsymbol{\xi}_{h}=\frac{\partial \phi}{\partial t}$, then the structure terms on the left hand side satisfies

$$
\begin{aligned}
& \rho_{s}\left(\frac{\partial^{2} \phi}{\partial t^{2}}, \frac{\partial \phi}{\partial t}\right)_{\Omega^{s}}+2 \nu_{s}\left(\mathbf{D}(\phi), \mathbf{D}\left(\frac{\partial \phi}{\partial t}\right)\right)_{\Omega^{s}}+\lambda\left(\nabla \cdot \phi, \nabla \cdot \frac{\partial \phi}{\partial t}\right)_{\Omega^{s}} \\
= & \frac{\partial}{\partial t}\left[\frac{\rho_{s}}{2}\left\|\frac{\partial \phi}{\partial t}\right\|_{0, \Omega^{s}}^{2}+\nu_{s}\|\mathbf{D}(\phi)\|_{0, \Omega^{s}}^{2}+\frac{\lambda}{2}\|\nabla \cdot \phi\|_{0, \Omega^{s}}^{2}\right] .
\end{aligned}
$$

By Cauchy-Schwartz and Young's inequalities, the structure part on the right hand side of (4.16) is bounded as

$$
\begin{aligned}
& \rho_{s}\left(\frac{\partial^{2} \boldsymbol{\psi}}{\partial t^{2}}, \frac{\partial \boldsymbol{\phi}}{\partial t}\right)_{\Omega^{s}}+2 \nu_{s}\left(\mathbf{D}(\boldsymbol{\psi}), \mathbf{D}\left(\frac{\partial \boldsymbol{\phi}}{\partial t}\right)\right)_{\Omega^{s}}+\lambda\left(\nabla \cdot \boldsymbol{\psi}, \nabla \cdot \frac{\partial \boldsymbol{\phi}}{\partial t}\right)_{\Omega^{s}} \\
\leq & \frac{\rho_{s}}{2}\left(\left\|\frac{\partial^{2} \boldsymbol{\psi}}{\partial t^{2}}\right\|_{0, \Omega^{s}}^{2}+\left\|\frac{\partial \phi}{\partial t}\right\|_{0, \Omega^{s}}^{2}\right)+2 \nu_{s}\left(\mathbf{D}(\boldsymbol{\psi}), \mathbf{D}\left(\frac{\partial \boldsymbol{\phi}}{\partial t}\right)\right)_{\Omega^{s}}+\lambda\left(\nabla \cdot \boldsymbol{\psi}, \nabla \cdot \frac{\partial \boldsymbol{\phi}}{\partial t}\right)_{\Omega^{s}}
\end{aligned}
$$

Setting $\mathbf{v}_{h}=\mathbf{I}$ in $(4.16)$,

$$
\left(\left.\frac{\partial \mathbf{I}}{\partial t}\right|_{\mathbf{y}}, \mathbf{I}\right)_{\Omega_{t}^{f}}=\frac{1}{2} \frac{\partial}{\partial t}\|\mathbf{I}\|_{0, \Omega_{t}^{f}}^{2}-\frac{1}{2}\left(\mathbf{I} \nabla \cdot \mathbf{z}_{h}, \mathbf{I}\right)_{\Omega_{t}^{f}}
$$

as shown in (4.7). Using (2.11), (4.19), Poincare inequality and the fact that $\left(\tilde{p}_{h}-p_{h}, \nabla \cdot \mathbf{I}\right)=$ $0, \theta\left(\mathbf{u}_{h}, \mathbf{I}, \mathbf{I}\right)_{\Omega_{t}^{f}}=\theta\left(\mathbf{z}_{h}, \mathbf{I}, \mathbf{I}\right)_{\Omega_{t}^{f}}=0$, we obtain a lower bound for the fluid terms on the left hand side of (4.16),

$$
\begin{aligned}
& \rho_{f}\left(\left.\frac{\partial \mathbf{I}}{\partial t}\right|_{\mathbf{y}}, \mathbf{I}\right)_{\Omega_{t}^{f}}+\rho_{f} \theta\left(\mathbf{u}_{h}, \mathbf{I}, \mathbf{I}\right)_{\Omega_{t}^{f}}+\rho_{f}\left[\frac{1}{2}\left(\mathbf{I} \nabla \cdot \mathbf{z}_{h}, \mathbf{I}\right)_{\Omega_{t}^{f}}-\theta\left(\mathbf{z}_{h}, \mathbf{I}, \mathbf{I}\right)_{\Omega_{t}^{f}}\right] \\
& +\left(\nu_{f}\left(\left|\mathbf{D}\left(\tilde{\mathbf{u}}_{h}\right)\right|\right) \mathbf{D}\left(\tilde{\mathbf{u}}_{h}\right), \mathbf{D}(\mathbf{I})\right)_{\Omega_{t}^{f}}-\left(\nu_{f}\left(\left|\mathbf{D}\left(\mathbf{u}_{h}\right)\right|\right) \mathbf{D}\left(\mathbf{u}_{h}\right), \mathbf{D}(\mathbf{I})\right)_{\Omega_{t}^{f}} \\
& -\left(\tilde{p}_{h}-p_{h}, \nabla \cdot \mathbf{I}\right)_{\Omega_{t}^{f}} \\
\geq & \frac{\rho_{f}}{2} \frac{\partial}{\partial t}\|\mathbf{I}\|_{0, \Omega_{t}^{f}}^{2}+K_{1}\|\mathbf{D}(\mathbf{I})\|_{0, \Omega_{t}^{f}}^{2} .
\end{aligned}
$$

Poincaré inequality and Young's inequality imply

$$
\begin{aligned}
& \rho_{f}\left(\left.\frac{\partial \mathbf{g}}{\partial t}\right|_{\mathbf{y}}, \mathbf{I}\right)_{\Omega_{t}^{f}}-\left(\tilde{p}_{h}-p, \nabla \cdot \mathbf{I}\right)_{\Omega_{t}^{f}} \\
\leq & C\left(\epsilon_{1}\right)\left(\left\|\left.\frac{\partial \mathbf{g}}{\partial t}\right|_{\mathbf{y}}\right\|_{0, \Omega_{t}^{f}}^{2}+\left\|\tilde{p}_{h}-p\right\|_{0, \Omega_{t}^{f}}^{2}\right)+\epsilon_{1}\|D(\mathbf{I})\|_{0, \Omega_{t}^{f}}^{2} .
\end{aligned}
$$

The estimate (3.17), Young's inequality and the stability result (4.6) imply

$$
\begin{aligned}
\rho_{f} \theta\left(\mathbf{u}_{h}, \mathbf{g}, \mathbf{I}\right)_{\Omega_{t}^{f}} \leq C\left\|\mathbf{u}_{h}\right\|_{0, \Omega_{t}^{f}}^{1 / 2}\left\|\mathbf{D}\left(\mathbf{u}_{h}\right)\right\|_{0, \Omega_{t}^{f}}^{1 / 2}\|\mathbf{D}(\mathbf{g})\|_{0, \Omega_{t}^{f}}\|\mathbf{D}(\mathbf{I})\|_{0, \Omega_{t}^{f}} \\
\leq C\left(\epsilon_{1}\right)\left\|\mathbf{D}\left(\mathbf{u}_{h}\right)\right\|_{0, \Omega_{t}^{f}}\|\mathbf{D}(\mathbf{g})\|_{0, \Omega_{t}^{f}}^{2}+\epsilon_{1}\|\mathbf{D}(\mathbf{I})\|_{0, \Omega_{t}^{f}}^{2} .
\end{aligned}
$$


The inequality (3.16) and Young's inequality imply that

$$
\begin{aligned}
\rho_{f} \theta(\mathbf{g}, \mathbf{u}, \mathbf{I})_{\Omega_{t}^{f}} & \leq C\|\mathbf{D}(\mathbf{u})\|_{0, \Omega_{t}^{f}}\|\mathbf{D}(\mathbf{g})\|_{0, \Omega_{t}^{f}}\|\mathbf{D}(\mathbf{I})\|_{0, \Omega_{t}^{f}} \\
& \leq C\left(\epsilon_{1}\right)\|\mathbf{D}(\mathbf{u})\|_{0, \Omega_{t}^{f}}^{2}\|\mathbf{D}(\mathbf{g})\|_{0, \Omega_{t}^{f}}^{2}+\epsilon_{1}\|\mathbf{D}(\mathbf{I})\|_{0, \Omega_{t}^{f}}^{2} .
\end{aligned}
$$

Also, (3.17) and the inequality $a b \leq \epsilon a^{\frac{4}{3}}+C(\epsilon) b^{4}$ imply

$$
\begin{aligned}
-\rho_{f} \theta(\mathbf{I}, \mathbf{u}, \mathbf{I})_{\Omega_{t}^{f}} & \leq C\|\mathbf{I}\|_{0, \Omega_{t}^{f}}^{\frac{1}{2}}\|\mathbf{D}(\mathbf{I})\|_{0, \Omega_{t}^{f}}^{\frac{3}{2}}\|\mathbf{D}(\mathbf{u})\|_{0, \Omega_{t}^{f}} \\
& \leq C\left(\epsilon_{1}\right)\|\mathbf{I}\|_{0, \Omega_{t}^{f}}^{2}\|\mathbf{D}(\mathbf{u})\|_{0, \Omega_{t}^{f}}^{4}+\epsilon_{1}\|\mathbf{D}(\mathbf{I})\|_{0, \Omega_{t}^{f}}^{2} .
\end{aligned}
$$

By (3.16) and (4.3) we have

$$
\begin{aligned}
\frac{1}{2}\left(\mathbf{g} \nabla \cdot \mathbf{z}_{h}, \mathbf{I}\right)_{\Omega_{t}^{f}}-\theta\left(\mathbf{z}_{h}, \mathbf{g}, \mathbf{I}\right)_{\Omega_{t}^{f}} & \leq C\left\|\mathbf{z}_{h}\right\|_{1, \infty, \Omega_{t}}\|\mathbf{D}(\mathbf{I})\|_{0, \Omega_{t}}\|\mathbf{D}(\mathbf{g})\|_{0, \Omega_{t}} \\
& \leq C\left(\epsilon_{1}\right)\|\mathbf{D}(\mathbf{g})\|_{0, \Omega_{t}^{f}}^{2}+\epsilon_{1}\|\mathbf{D}(\mathbf{I})\|_{0, \Omega_{t}^{f}}^{2} .
\end{aligned}
$$

The bound of the viscosity term is determined by (2.13) as

$$
\begin{aligned}
& \left(\nu_{f}\left(\left|\mathbf{D}\left(\tilde{\mathbf{u}}_{h}\right)\right|\right) \mathbf{D}\left(\tilde{\mathbf{u}}_{h}\right), \mathbf{D}(\mathbf{I})\right)_{\Omega_{t}^{f}}-\left(\nu_{f}(|\mathbf{D}(\mathbf{u})|) \mathbf{D}(\mathbf{u}), \mathbf{D}(\mathbf{I})\right)_{\Omega_{t}^{f}} \\
\leq & K_{3}\|\mathbf{D}(\mathbf{g})\|_{0, \Omega_{t}^{f}}\|\mathbf{D}(\mathbf{I})\|_{0, \Omega_{t}^{f}} \leq C\left(\epsilon_{1}\right)\|\mathbf{D}(\mathbf{g})\|_{0, \Omega_{t}^{f}}^{2}+\epsilon_{1}\|\mathbf{D}(\mathbf{I})\|_{0, \Omega_{t}^{f}}^{2} .
\end{aligned}
$$

Using the estimates (4.21) - (4.26), a bound of the fluid part in the right hand side of (4.16) can be obtained as

$$
\begin{aligned}
\rho_{f}\left(\left.\frac{\partial \mathbf{g}}{\partial t}\right|_{\mathbf{y}}, \mathbf{I}\right)_{\Omega_{t}^{f}}+\rho_{f}\left[\theta\left(\mathbf{u}_{h}, \mathbf{g}, \mathbf{I}\right)_{\Omega_{t}^{f}}+\theta(\mathbf{g}, \mathbf{u}, \mathbf{I})_{\Omega_{t}^{f}}-\theta(\mathbf{I}, \mathbf{u}, \mathbf{I})_{\Omega_{t}^{f}}\right] \\
+\rho_{f}\left[\frac{1}{2}\left(\mathbf{g} \nabla \cdot \mathbf{z}_{h}, \mathbf{I}\right)_{\Omega_{t}^{f}}-\theta\left(\mathbf{z}_{h}, \mathbf{g}, \mathbf{I}\right)_{\Omega_{t}^{f}}\right] \\
+\left(\nu_{f}\left(\left|\mathbf{D}\left(\tilde{\mathbf{u}}_{h}\right)\right|\right) \mathbf{D}\left(\tilde{\mathbf{u}}_{h}\right), \mathbf{D}(\mathbf{I})\right)_{\Omega_{t}^{f}}-\left(\nu_{f}(|\mathbf{D}(\mathbf{u})|) \mathbf{D}(\mathbf{u}), \mathbf{D}(\mathbf{I})\right)_{\Omega_{t}^{f}}-\left(\tilde{p}_{h}-p, \nabla \cdot \mathbf{I}\right)_{\Omega_{t}^{f}} \\
\leq \quad C\left(\epsilon_{1}\right)\left[\left\|\left.\frac{\partial \mathbf{g}}{\partial t}\right|_{\mathbf{y}}\right\|_{0, \Omega_{t}^{f}}^{2}+\left\|\mathbf{D}\left(\mathbf{u}_{h}\right)\right\|_{0, \Omega_{t}^{f}}\|\mathbf{D}(\mathbf{g})\|_{0, \Omega_{t}^{f}}^{2}+\|\mathbf{D}(\mathbf{u})\|_{0, \Omega_{t}^{f}}^{2}\|\mathbf{D}(\mathbf{g})\|_{0, \Omega_{t}^{f}}^{2}\right. \\
\left.+\|\mathbf{I}\|_{0, \Omega_{t}^{f}}^{2}\|\mathbf{D}(\mathbf{u})\|_{0, \Omega_{t}^{f}}^{4}+\|\mathbf{D}(\mathbf{g})\|_{0, \Omega_{t}^{f}}^{2}+\left\|\tilde{p}_{h}-p\right\|_{0, \Omega_{t}^{f}}^{2}\right]+6 \epsilon_{1}\|\mathbf{D}(\mathbf{I})\|_{0, \Omega_{t}^{f}}^{2} .
\end{aligned}
$$

Setting $\epsilon_{1}=\frac{K_{1}}{12}$, using (4.17), (4.18), (4.20), (4.27) and multiplying both sides by $2,(4.16)$ implies

$$
\begin{gathered}
\frac{\partial}{\partial t}\left[\rho_{s}\left\|\frac{\partial \phi}{\partial t}\right\|_{0, \Omega^{s}}^{2}+2 \nu_{s}\|\mathbf{D}(\boldsymbol{\phi})\|_{0, \Omega^{s}}^{2}+\lambda\|\nabla \cdot \boldsymbol{\phi}\|_{0, \Omega^{s}}^{2}+\rho_{f}\|\mathbf{I}\|_{0, \Omega_{t}^{f}}^{2}\right]+K_{1}\|\mathbf{D}(\mathbf{I})\|_{0, \Omega_{t}^{f}}^{2} \\
\leq \rho_{s}\left\|\frac{\partial^{2} \boldsymbol{\psi}}{\partial t^{2}}\right\|_{0, \Omega^{s}}^{2}+\rho_{s}\left\|\frac{\partial \boldsymbol{\phi}}{\partial t}\right\|_{0, \Omega^{s}}^{2}+4 \nu_{s}\left(\mathbf{D}(\boldsymbol{\psi}), \mathbf{D}\left(\frac{\partial \boldsymbol{\phi}}{\partial t}\right)\right)_{\Omega^{s}}+2 \lambda\left(\nabla \cdot \boldsymbol{\psi}, \nabla \cdot \frac{\partial \boldsymbol{\phi}}{\partial t}\right)_{\Omega^{s}} \\
+C\left[\left\|\left.\frac{\partial \mathbf{g}}{\partial t}\right|_{\mathbf{y}}\right\|_{0, \Omega_{t}^{f}}^{2}+\left\|\mathbf{D}\left(\mathbf{u}_{h}\right)\right\|_{0, \Omega_{t}^{f}}\|\mathbf{D}(\mathbf{g})\|_{0, \Omega_{t}^{f}}^{2}+\|\mathbf{D}(\mathbf{u})\|_{0, \Omega_{t}^{f}}^{2}\|\mathbf{D}(\mathbf{g})\|_{0, \Omega_{t}^{f}}^{2}\right. \\
\left.+\|\mathbf{I}\|_{0, \Omega_{t}^{f}}^{2}\|\mathbf{D}(\mathbf{u})\|_{0, \Omega_{t}^{f}}^{4}+\|\mathbf{D}(\mathbf{g})\|_{0, \Omega_{t}^{f}}^{2}+\left\|\tilde{p}_{h}-p\right\|_{0, \Omega_{t}^{f}}^{2}\right] .
\end{gathered}
$$


Assuming $\mathbf{u} \in L^{4}\left(0, T ; \mathbf{H}^{1}\left(\Omega_{t}^{f}\right)\right)$, and using the same technique shown in [14] (p.157), which is equivalent to the Gronwall's inequality, we have

$$
\begin{aligned}
& \rho_{s}\left\|\frac{\partial \phi}{\partial t}\right\|_{0, \Omega^{s}}^{2}+2 \nu_{s}\|\mathbf{D}(\boldsymbol{\phi})\|_{0, \Omega^{s}}^{2}+\lambda\|\nabla \cdot \boldsymbol{\phi}\|_{0, \Omega^{s}}^{2}+\rho_{f}\|\mathbf{I}\|_{0, \Omega_{t}^{f}}^{2}+K_{1} \int_{0}^{t}\|\mathbf{D}(\mathbf{I})\|_{0, \Omega_{\tilde{t}}^{f}}^{2} d \tilde{t} \\
\leq & C\left(\rho_{s}\left\|\frac{\partial \boldsymbol{\phi}(0)}{\partial t}\right\|_{0, \Omega^{s}}^{2}+2 \nu_{s}\|\mathbf{D}(\boldsymbol{\phi}(0))\|_{0, \Omega^{s}}^{2}+\lambda\|\nabla \cdot \boldsymbol{\phi}(0)\|_{0, \Omega^{s}}^{2}+\rho_{f}\|\mathbf{I}(0)\|_{0, \Omega_{0}^{f}}^{2}\right) \\
+ & \frac{\rho_{s}}{2} \int_{0}^{t}\left\|\frac{\partial \boldsymbol{\phi}}{\partial \tilde{t}}\right\|_{0, \Omega^{s}}^{2} d \tilde{t}+\int_{0}^{t} 4 \nu_{s}\left(\mathbf{D}(\boldsymbol{\psi}), \mathbf{D}\left(\frac{\partial \boldsymbol{\phi}}{\partial \tilde{t}}\right)\right)_{\Omega^{s}} d \tilde{t}+\int_{0}^{t} 2 \lambda\left(\nabla \cdot \boldsymbol{\psi}, \nabla \cdot \frac{\partial \boldsymbol{\phi}}{\partial \tilde{t}}\right)_{\Omega^{s}} d \tilde{t} \\
+ & C \int_{0}^{t}\left[\left\|\frac{\partial^{2} \boldsymbol{\psi}}{\partial \tilde{t}^{2}}\right\|_{0, \Omega^{s}}^{2}+\left\|\left.\frac{\partial \mathbf{g}}{\partial \tilde{t}}\right|_{\mathbf{y}}\right\|_{0, \Omega_{\tilde{t}}^{f}}^{2}+\left\|\mathbf{D}\left(\mathbf{u}_{h}\right)\right\|_{0, \Omega_{\tilde{t}}^{f}}\|\mathbf{D}(\mathbf{g})\|_{0, \Omega_{\tilde{t}}^{f}}^{2}\right. \\
& \left.+\|\mathbf{D}(\mathbf{u})\|_{0, \Omega_{\tilde{t}}^{f}}^{2}\|\mathbf{D}(\mathbf{g})\|_{0, \Omega_{\tilde{t}}^{f}}^{2}+\|\mathbf{D}(\mathbf{g})\|_{0, \Omega_{\tilde{t}}^{f}}^{2}+\left\|\tilde{p}_{h}-p\right\|_{0, \Omega_{\tilde{t}}^{f}}^{2}\right] d \tilde{t} .
\end{aligned}
$$

Using integration by parts,

$$
\begin{aligned}
& \int_{0}^{t} 4 \nu_{s}\left(\mathbf{D}(\psi), \mathbf{D}\left(\frac{\partial \phi}{\partial \tilde{t}}\right)\right)_{\Omega^{s}} d \tilde{t} \\
= & 4 \nu_{s} \int_{\Omega^{s}} \int_{0}^{t} \mathbf{D}(\boldsymbol{\psi}) \mathbf{D}\left(\frac{\partial \phi}{\partial \tilde{t}}\right) d \tilde{t} d \Omega^{s} \\
= & 4 \nu_{s} \int_{\Omega^{s}}\left[\mathbf{D}(\boldsymbol{\psi}(t)) \mathbf{D}(\phi(t))-\mathbf{D}(\boldsymbol{\psi}(0)) \mathbf{D}(\phi(0))-\int_{0}^{t} \mathbf{D}\left(\frac{\partial \boldsymbol{\psi}}{\partial \tilde{t}}\right) \mathbf{D}(\phi) d \tilde{t}\right] d \Omega^{s},
\end{aligned}
$$

and Cauchy-Schwartz and Young's inequalities yield that

$$
\begin{aligned}
\int_{0}^{t} 4 \nu_{s}(\mathbf{D}(\boldsymbol{\psi}), & \left.\mathbf{D}\left(\frac{\partial \boldsymbol{\phi}}{\partial \tilde{t}}\right)\right)_{\Omega^{s}} d \tilde{t} \leq 4 C\left(\epsilon_{2}\right) \nu_{s}\|\mathbf{D}(\boldsymbol{\psi})\|_{0, \Omega^{s}}^{2}+4 \epsilon_{2} \nu_{s}\|\mathbf{D}(\boldsymbol{\phi})\|_{0, \Omega^{s}}^{2} \\
& +2 \nu_{s}\left(\|\mathbf{D}(\boldsymbol{\psi}(0))\|_{0, \Omega^{s}}^{2}+\|\mathbf{D}(\boldsymbol{\phi}(0))\|_{0, \Omega^{s}}^{2}\right) \\
& +\int_{0}^{t}\left(4 C\left(\epsilon_{2}\right) \nu_{s}\left\|\mathbf{D}\left(\frac{\partial \boldsymbol{\psi}}{\partial \tilde{t}}\right)\right\|_{0, \Omega^{s}}^{2}+4 \epsilon_{2} \nu_{s}\|\mathbf{D}(\boldsymbol{\phi})\|_{0, \Omega^{s}}^{2}\right) d \tilde{t}
\end{aligned}
$$

Similarly, we have

$$
\begin{aligned}
\int_{0}^{t} 2 \lambda(\nabla \cdot \boldsymbol{\psi}, & \left.\nabla \cdot \frac{\partial \boldsymbol{\phi}}{\partial \tilde{t}}\right)_{\Omega^{s}} d \tilde{t} \leq 2 C\left(\epsilon_{3}\right) \lambda\|\nabla \cdot \boldsymbol{\psi}\|_{0, \Omega^{s}}^{2}+2 \epsilon_{3} \lambda\|\nabla \cdot \boldsymbol{\phi}\|_{0, \Omega^{s}}^{2} \\
& +\lambda\left(\|\nabla \cdot \boldsymbol{\psi}(0)\|_{0, \Omega^{s}}^{2}+\|\nabla \cdot \boldsymbol{\phi}(0)\|_{0, \Omega^{s}}^{2}\right) \\
& +\int_{0}^{t}\left(2 C\left(\epsilon_{3}\right) \lambda\left\|\nabla \cdot \frac{\partial \boldsymbol{\psi}}{\partial \tilde{t}}\right\|_{0, \Omega^{s}}^{2}+2 \epsilon_{3} \lambda\|\nabla \cdot \boldsymbol{\phi}\|_{0, \Omega^{s}}^{2}\right) d \tilde{t} .
\end{aligned}
$$

Hölder inequality implies

$$
\int_{0}^{t}\left\|\mathbf{D}\left(\mathbf{u}_{h}\right)\right\|_{0, \Omega_{\tilde{t}}^{f}}\|\mathbf{D}(\mathbf{g})\|_{0, \Omega_{\tilde{t}}^{f}}^{2} d \tilde{t} \leq C\left\|\mathbf{D}\left(\mathbf{u}_{h}\right)\right\|_{L^{2}\left(0, T ; L^{2}\right)}^{2}\|\mathbf{D}(\mathbf{g})\|_{L^{4}\left(0, T ; L^{2}\right)}^{4},
$$


where $\left\|\mathbf{D}\left(\mathbf{u}_{h}\right)\right\|_{L^{2}\left(0, T ; L^{2}\right)}^{2}$ is bounded by the stability result (4.6). Also,

$$
\begin{aligned}
\int_{0}^{t}\|\mathbf{D}(\mathbf{u})\|_{0, \Omega_{\tilde{t}}^{f}}^{2}\|\mathbf{D}(\mathbf{g})\|_{0, \Omega_{\tilde{t}}^{f}}^{2} d \tilde{t} & \leq C\|\mathbf{D}(\mathbf{u})\|_{L^{4}\left(0, T ; L^{2}\right)}^{4}\|\mathbf{D}(\mathbf{g})\|_{L^{4}\left(0, T ; L^{2}\right)}^{4} \\
& \leq C\|\mathbf{D}(\mathbf{g})\|_{L^{4}\left(0, T ; L^{2}\right)}^{4},
\end{aligned}
$$

since $\mathbf{u} \in L^{4}\left(0, T ; \mathbf{H}^{1}\left(\Omega_{t}^{f}\right)\right)$.

Setting $\epsilon_{2}=\epsilon_{3}=\frac{1}{4}$, applying (4.31) - (4.34) and using the fact $\|\nabla \cdot \boldsymbol{\xi}\|_{0, \Omega^{s}} \leq C\|\nabla \boldsymbol{\xi}\|_{0, \Omega^{s}}$ for $\boldsymbol{\xi} \in \mathbf{H}^{1}\left(\Omega^{s}\right)$, (4.29) implies that

$$
\begin{aligned}
& \rho_{s}\left\|\frac{\partial \boldsymbol{\phi}}{\partial t}\right\|_{0, \Omega^{s}}^{2}+\nu_{s}\|\mathbf{D}(\boldsymbol{\phi})\|_{0, \Omega^{s}}^{2}+\frac{\lambda}{2}\|\nabla \cdot \boldsymbol{\phi}\|_{0, \Omega^{s}}^{2}+\rho_{f}\|\mathbf{I}\|_{0, \Omega_{t}^{f}}^{2}+K_{1} \int_{0}^{t}\|\mathbf{D}(\mathbf{I})\|_{0, \Omega_{\tilde{t}}^{f}}^{2} d \tilde{t} \\
\leq & C\left(\left\|\frac{\partial \boldsymbol{\phi}(0)}{\partial t}\right\|_{0, \Omega^{s}}^{2}+\|\mathbf{D}(\boldsymbol{\phi}(0))\|_{0, \Omega^{s}}^{2}+\|\nabla \cdot \boldsymbol{\phi}(0)\|_{0, \Omega^{s}}^{2}+\|\mathbf{I}(0)\|_{0, \Omega_{0}^{f}}^{2}+\|\mathbf{D}(\boldsymbol{\psi}(0))\|_{0, \Omega^{s}}^{2}\right) \\
& +\int_{0}^{t}\left(\frac{\rho_{s}}{2}\left\|\frac{\partial \boldsymbol{\phi}}{\partial \tilde{t}}\right\|_{0, \Omega^{s}}^{2}+\nu_{s}\|\mathbf{D}(\boldsymbol{\phi})\|_{0, \Omega^{s}}^{2}+\frac{\lambda}{2}\|\nabla \cdot \boldsymbol{\phi}\|_{0, \Omega^{s}}^{2}\right) d \tilde{t} \\
& +C \int_{0}^{t}\left\|\frac{\partial^{2} \boldsymbol{\psi}}{\partial \tilde{t}^{2}}\right\|_{0, \Omega^{s}}^{2}+\left\|\left.\frac{\partial \mathbf{g}}{\partial \tilde{t}}\right|_{\mathbf{y}}\right\|_{0, \Omega_{\tilde{t}}^{f}}^{2}+\|\mathbf{D}(\mathbf{g})\|_{0, \Omega_{\tilde{t}}^{f}}^{2}+\left\|\tilde{p}_{h}-p\right\|_{0, \Omega_{\tilde{t}}^{f}}^{2}+\left\|\mathbf{D}\left(\frac{\partial \boldsymbol{\psi}}{\partial \tilde{t}}\right)\right\|_{0, \Omega^{s}}^{2} d \tilde{t} \\
& +C\left(\|\mathbf{D}(\mathbf{g})\|_{L^{4}\left(0, T ; L^{2}\right)}^{4}+\|\mathbf{D}(\psi)\|_{0, \Omega^{s}}^{2}\right) .
\end{aligned}
$$

The stated error estimate can be obtained applying Gronwall's lemma, the triangular inequality and the inf-sup condition (4.1).

\section{$5 \quad$ Numerical results}

Numerical tests were carried out on a non-physical problem. Although the previous analysis is based on the general quasi-Newtonian fluid involving a general viscosity function $\nu(|\mathbf{D}(\mathbf{u})|)$, we consider a specific case, the Cross model, as the quasi-Newtonian flow for numerical tests. The viscosity function of the Cross model is given by

$$
\nu_{f}(|\mathbf{D}(\mathbf{u})|):=\nu_{\infty}+\frac{\left(\nu_{0}-\nu_{\infty}\right)}{1+(\kappa|\mathbf{D}(\mathbf{u})|)^{2-r}}
$$

where $\kappa>0$ is a time constant, $1 \leq r \leq 2$ is a dimensionless rate constant, and $\nu_{\infty}$ and $\nu_{0}$ denote limiting viscosity values at an infinite and zero shear rate, respectively, assumed to satisfy $0 \leq \nu_{\infty} \leq \nu_{0}$. Throughout this section, we will restrict our focus to the case where $\kappa=1, \nu_{0}=1$ and $\nu_{\infty}=0.5$. Note that with the choice of $r=2$, the fluid becomes Newtonian.

Since we are interested in the convergence results of the FSI problem, we make the same assumption as in [1]: the system has infinitesimal displacements of the fluid domain 
and the structure, but with non-negligible velocity of the interface. Parameters chosen for the simulations are: $\rho_{f}=1.0, \rho_{s}=1.9 ; \nu_{s}=3$ and $\lambda=4.5$. Initial conditions, body forces, and boundary conditions are appropriately given so that the exact solution on the computational domain $\Omega^{f}=[0,1] \times[0,1]$ and $\Omega^{s}=[0,1] \times[0,1]$ is

$$
\begin{aligned}
\mathbf{u}_{1}= & \cos (x+t) \sin (y+t)+\sin (x+t) \cos (y+t), \\
\mathbf{u}_{2}= & -\sin (x+t) \cos (y+t)-\cos (x+t) \sin (y+t), \\
p= & \left(0.5+\frac{0.5}{1+(\sin (x+t) \sin (y+t)-\cos (x+t) \cos (y+t))^{4-2 r}}\right) \\
& \cdot(\sin (x+t) \sin (y+t)-\cos (x+t) \cos (y+t) \\
& +2 \nu_{s} \cos (x+t) \sin (y+t), \\
\boldsymbol{\eta}_{1}= & \sin (x+t) \sin (y+t), \\
\boldsymbol{\eta}_{2}= & \cos (x+t) \cos (y+t) .
\end{aligned}
$$

We performed simulations over one time step to check convergence rates. The TaylorHood pair $\left(\mathbb{Q}_{2}, \mathbb{Q}_{1}\right)$ was used to solve the fluid equations, while $\mathbb{Q}_{2}$ finite elements were used for the structure displacement. For the spatial convergence tests, we set $\Delta t=1 e-10 \mathrm{~s}$ and used uniform meshes. All computations were performed using the deal.II library.

We set $r=2$ in the Cross model for the first convergence test, which is equivalent to a linear Newtonian fluid-structure system. The FSI problem was solved with a sequence of decreasing mesh size, and the results are presented in Table 1 and Table 2. We obtained the theoretical spatial convergence rate at which the computed solution converges upon the true solution.

\begin{tabular}{|c|c|c|c|c|}
\hline \hline $\mathrm{h}$ & $\left\|\mathbf{u}^{n}-\mathbf{u}^{\text {true }}\right\|_{L^{2}}$ & Rate & $\left\|\mathbf{u}^{n}-\mathbf{u}^{\text {true }}\right\|_{H^{1}}$ & Rate \\
\hline $1 / 8$ & $2.6687 \mathrm{e}-005$ & - & $3.5233 \mathrm{e}-003$ & - \\
\hline $1 / 16$ & $4.0229 \mathrm{e}-006$ & 2.73 & $1.0476 \mathrm{e}-003$ & 1.75 \\
\hline $1 / 32$ & $5.4278 \mathrm{e}-007$ & 2.89 & $2.8861 \mathrm{e}-004$ & 1.86 \\
\hline $1 / 64$ & $6.7389 \mathrm{e}-008$ & 3.01 & $7.2660 \mathrm{e}-005$ & 1.99 \\
\hline
\end{tabular}

Table 1: fluid convergence result for linear FSI

\begin{tabular}{|c|c|c|c|c|}
\hline \hline $\mathrm{h}$ & $\left\|\boldsymbol{\eta}^{n}-\boldsymbol{\eta}^{\text {true }}\right\|_{L^{2}}$ & Rate & $\left\|\boldsymbol{\eta}^{n}-\boldsymbol{\eta}^{\text {true }}\right\|_{H^{1}}$ & Rate \\
\hline $1 / 8$ & $9.8795 \mathrm{e}-006$ & - & $1.0112 \mathrm{e}-003$ & - \\
\hline $1 / 16$ & $4.0229 \mathrm{e}-006$ & 2.70 & $2.9652 \mathrm{e}-004$ & 1.77 \\
\hline $1 / 32$ & $2.0234 \mathrm{e}-007$ & 2.91 & $9.0566 \mathrm{e}-005$ & 1.88 \\
\hline $1 / 64$ & $2.4947 \mathrm{e}-008$ & 3.02 & $2.004 \mathrm{e}-005$ & 2.01 \\
\hline
\end{tabular}

Table 2: structure convergence result for linear FSI

We considered a nonlinear quasi-Newtonian case for the second convergence test by setting $r=1.5$. Similar simulations have been done as the first test, and the results are presented in Table 3 and Table 4. Again, we obtained the theoretical convergence rate upon the true solution, and observed no significant difference between the Newtonian and non-Newtonian cases. 


\begin{tabular}{|c|c|c|c|c|}
\hline \hline $\mathrm{h}$ & $\left\|\mathbf{u}^{n}-\mathbf{u}^{\text {true }}\right\|_{L^{2}}$ & Rate & $\left\|\mathbf{u}^{n}-\mathbf{u}^{\text {true }}\right\|_{H^{1}}$ & Rate \\
\hline $1 / 8$ & $5.7862 \mathrm{e}-005$ & - & $4.1652 \mathrm{e}-003$ & - \\
\hline $1 / 16$ & $7.8783 \mathrm{e}-006$ & 2.88 & $1.2299 \mathrm{e}-003$ & 1.76 \\
\hline $1 / 32$ & $9.9565 . \mathrm{e}-007$ & 2.98 & $3.5675 \mathrm{e}-004$ & 1.79 \\
\hline $1 / 64$ & $1.1943 \mathrm{e}-007$ & 3.06 & $8.7673 \mathrm{e}-005$ & 2.02 \\
\hline
\end{tabular}

Table 3: fluid convergence result for non-linear FSI

\begin{tabular}{|c|c|c|c|c|}
\hline \hline $\mathrm{h}$ & $\left\|\boldsymbol{\eta}^{n}-\boldsymbol{\eta}^{\text {true }}\right\|_{L^{2}}$ & Rate & $\left\|\boldsymbol{\eta}^{n}-\boldsymbol{\eta}^{\text {true }}\right\|_{H^{1}}$ & Rate \\
\hline $1 / 8$ & $1.5452 \mathrm{e}-005$ & - & $1.8293 \mathrm{e}-003$ & - \\
\hline $1 / 16$ & $2.2872 \mathrm{e}-006$ & 2.76 & $5.9114 \mathrm{e}-004$ & 1.63 \\
\hline $1 / 32$ & $3.1872 \mathrm{e}-007$ & 2.84 & $1.5769 \mathrm{e}-004$ & 1.91 \\
\hline $1 / 64$ & $3.4989 \mathrm{e}-008$ & 3.19 & $3.9066 \mathrm{e}-005$ & 2.01 \\
\hline
\end{tabular}

Table 4: structure convergence result for non-linear FSI

\section{Conclusion}

We considered a monolithic formulation for the quasi-Newtonian fluid-structure interaction problem. An Arbitrary Lagrangian Eulerian mapping was introduced to deal with the time derivative term in the fluid equation, and the two dynamic equations were combined into one formulation using the interface conditions. After defining finite element spaces, the finite element formulation was obtained for which we proved the stability and error estimate. Numerical tests were performed on both Newtonian and non-Newtoninan cases, and for both cases we obtained the theoretical convergence rates in $L_{2}$ and $H_{1}$ norms.

Future work will include more analysis for fully discretization formulation and corresponding numerical experiments.

\section{References}

[1] M. Astorino, C. Grandmont, Convergence analysis of a projection semi-implicit coupling scheme for fluid-structure interaction problems, Numer. Math., 116, 721-767, 2010 .

[2] T. Bodnár and A. Sequeira, Numerical study of the significance of the non-Netwonian nature of blood in steady flow through a stenosed vessel, Advances in Mathematical Fluid Mechanics, Springer, 83-104, 2010.

[3] T. Bodnár, A. Sequeira, and M. Prosi, On the shear-thinning and viscoelastic effects of blood flow under various flow rates, Appl. Math. Comput., 217, 5055-5067, 2011.

[4] D. Boffi and L. Gastaldi, Stability and geometric conservation laws for ALE formulations, Comput. Methods. Appl. Mech. Engrg., 193, 4717-4739, 2004. 
[5] P. Causin, J-F. Gerbeau and F. Nobile, Added-mass effect in the design of partitioned algorithms for fluid-structure problems, Comput. Methods Appl. Mech. Engrg. 194, 4506-4527, 2005.

[6] J. Donea, S. Giuliani, J.P. Halleux, An arbitrary Lagrangian-Eulerian finite element method for transient dynamic fluid-structure interactions, Comput. Methods Appl. Mech. Engrg., 33, 689-723, 1982.

[7] L. Formaggia, J.F. Gerbeau, F. Nobile, A. Quarteroni, On the Coupling of 3D and 1D Navier-Stokes equations for Flow Problems in Compliant Vessels, Comput. Methods Appl. Mech. Engrg., 191, 561-582, 2001.

[8] L. Formaggia and F. Nobile, A stability analysis for the arbitrary Lagrangian Eulerian formulation with finite elements, East-West J. Numer. Math., 7, 105-131, 1999.

[9] L. Formaggia, A. Moura and F. Nobile, On the stability of the coupling of 3D and 1D fluid-structure interaction models for blood flow simulations, Math. Mod. Numer. Anal., 41, 743-769, 2007.

[10] L. Gastaldi, A priori error estimates for the Arbitrary Lagrangian Eulerian formulation with finite elements, East-West J. Numer. Math., 9, 123-156, 2001.

[11] Y. He. The Euler implicit/explicit scheme for the 2D time-dependent Navier-Stokes equations with smooth or non-smooth initial data, Math. Comput., 77, 2097-2124. 2008 .

[12] T.J.R. Hughes, W.K. Liu and T.K. Zimmermann, Lagrangian-Eulerian finite element formulation for incompressible viscous flows, Comput. Methods. Appl. Mech. Engrg., 29, 329-349, 1981.

[13] J. Janela, A. Moura, and A. Sequeira, A 3D non-Newtonian fluid-structure interaction model for blood flow in arteries, J. Comput. Appl. Math., 234, 2783-2791, 2010.

[14] W. Layton, Introduction to the numerical analysis of incompressible viscous flows, SIAM, Philadelphia, 2008.

[15] H. Lee, Numerical approximation of Quasi-Newtoninan flows by ALE-FEM, Numerical Methods for PDEs, 28, 1667-1695, 2012.

[16] M. Lukáčová and A. Zauškozá, Numerical modelling of shear-thinning non-Newtonian flows in compliant vessels, Int. J. Numer. Meth. Fluids, 56, 1409-1415, 2008.

[17] J.S. Martin, L. Smaranda and T. Takahashi, Convergence of finite element/ALE method for the Stokes equations in a domain depending on time, Journal of Computational and Applied Mathematics, 230, 521-545, 2009.

[18] L. Nadau and A. Sequeira, Numerical simulations of shear dependent viscoelastic flows with a combined finite element-finite volume method, Comput. Math. Appl., 53, 547$568,2007$. 
[19] F. Nobile, Numerical approximation of fluid-structure interaction problems with application to haemodynamics, Ph.D. thesis, 2001.

[20] F. Nobile and C. Vergara, An effective fluid-structure interaction formulation for vascular dynamics by generalized Robin conditions, SIAM J. Sci. Comp., 30, 731-763, 2008 . 